\title{
Flows in Almost Linear Time via Adaptive Preconditioning
}

\author{
Rasmus Kyng* \\ Harvard University \\ Cambridge, USA \\ rjkyng@gmail.com \\ Sushant Sachdeva $\ddagger$
UToronto
Toronto, Canada
sachdeva@cs.toronto.edu
}

\begin{abstract}
We present algorithms for solving a large class of flow and regression problems on unit weighted graphs to $(1+1 / p o l y(n))$ accuracy in almost-linear time. These problems include $\ell_{p}$-norm minimizing flow for $p$ large $\left(p \in\left[\omega(1), o\left(\log ^{2 / 3} n\right)\right]\right)$, and their duals, $\ell_{p}$-norm semi-supervised learning for $p$ close to 1 .

As $p$ tends to infinity, $p$-norm flow and its dual tend to max-flow and min-cut respectively. Using this connection and our algorithms, we give an alternate approach for approximating undirected maxflow, and the first almost-linear time approximations of discretizations of total variation minimization objectives.

Our framework is inspired by the routing-based solver for Laplacian linear systems by Spielman and Teng (STOC '04, SIMAX '14), and is based on several new tools we develop, including adaptive non-linear preconditioning, tree-routings, and (ultra-)sparsification for mixed $\ell_{2}$ and $\ell_{p}$ norm objectives.
\end{abstract}

\section{CCS CONCEPTS}

- Mathematics of computing $\rightarrow$ Network flows; Convex optimization; • Theory of computation $\rightarrow$ Network flows; Sparsification and spanners; Convex optimization.

\section{KEYWORDS}

Network flows, convex optimization, graph sparsification, preconditioning

\section{ACM Reference Format:}

Rasmus Kyng, Richard Peng, Sushant Sachdeva, and Di Wang. 2019. Flows in Almost Linear Time via Adaptive Preconditioning. In Proceedings of the

*Supported by ONR grant N00014-18-1-2562.

$\dagger$ Supported in part by the National Science Foundation under Grant No. 1718533.

¥ Supported by the Natural Sciences and Engineering Research Council of Canada (NSERC), and a Connaught New Researcher award.

${ }^{\S}$ Supported in part by the National Science Foundation under Grant No. 1718533.

Permission to make digital or hard copies of all or part of this work for personal or classroom use is granted without fee provided that copies are not made or distributed for profit or commercial advantage and that copies bear this notice and the full citation on the first page. Copyrights for components of this work owned by others than ACM must be honored. Abstracting with credit is permitted. To copy otherwise, or republish, to post on servers or to redistribute to lists, requires prior specific permission and/or a fee. Request permissions from permissions@acm.org.

STOC '19, fune 23-26, 2019, Phoenix, AZ, USA

(c) 2019 Association for Computing Machinery.

ACM ISBN 978-1-4503-6705-9/19/06 . .\$15.00

https://doi.org/10.1145/3313276.3316410

\author{
Richard Peng ${ }^{\dagger}$ \\ Georgia Tech / MSR Redmond \\ Atlanta / Redmond, USA \\ richard.peng@gmail.com
}

\author{
Di Wang $§$ \\ Georgia Tech \\ Atlanta, USA \\ di.wang@cc.gatech.edu
}

51st Annual ACM SIGACT Symposium on the Theory of Computing (STOC '19), June 23-26, 2019, Phoenix, AZ, USA. ACM, New York, NY, USA, 12 pages. https://doi.org/10.1145/3313276.3316410

\section{INTRODUCTION}

Network flow problems in various forms play a central role in combinatorial optimization. A generic network flow problem can be succinctly formulated from a graph $G=(V, E)$ with edge-vertex incidence matrix $B$, a vector $\boldsymbol{b}$ indicating the required residues on vertices (satisfying $\mathbf{1}^{T} \boldsymbol{b}=0$ ), and a norm $p$ as finding a flow $f$ with demands $\boldsymbol{b}$ that minimize a specified norm $\|\cdot\|$ :

$$
\min _{B^{\top} f=b}\|f\| \text {. }
$$

When $\|\cdot\|=\|\cdot\|_{\infty}$, we get the classical maximum flow problem, which together with its dual, minimum $s-t$ cut, are some of the most well-studied and fundamental problems in the area [13, 21, 23, 26$30,33,52,60]$.

The maximum flow problem and its dual, minimum-cuts, as well as their extensions to minimum cost flows $[3,21]$ and parametric flows [9, 25] have wide ranges of applications [37, 46, 53]. The study of flow and cut problems has a long history of motivating broader developments in algorithms including the introduction of strongly polynomial time as a benchmark of algorithmic efficiency [20,21], the development of tree data structures [24, 64, 65], and randomized graph algorithms and graph approximations [10, 32].

A recent revolution in the design of graph algorithms was spurred by developments in the $\ell_{2}$-norm case of Problem (1), equivalent to electrical flows. The development of nearly-linear time highaccuracy solvers for Laplacian linear systems, which are the duals of electrical flows [36, 39, 42, 44, 67] led to improved algorithms for graph partitioning [51], random walks and spanning trees [34, 59], and somewhat surprisingly, drastic improvements in low-accuracy algorithms (i.e. algorithms for $(1+\epsilon)$-approximate solutions with running time scaling as poly $(1 / \epsilon)$ ) for undirected maximum flow and transshipment (the $\ell_{1}$ case of Problem (1)), yielding nearlylinear time algorithms [13, 35, 54, 61-63].

Progress on high accuracy algorithms (i.e. algorithms for $(1+\epsilon)$ approximate solutions with running time scaling as $\log (1 / \epsilon)$ for flow problems beyond electrical flows has been more limited. For general capacities, the best strongly polynomial time algorithms run in about quadratic time due to the flow decomposition barrier [21, $24,27,30,52]$. For graphs with polynomially bounded capacities, the 
current best running time is $\widetilde{O}(m \sqrt{n})$ due to Lee and Sidford [45]. On sparse graphs, such a bound can still be viewed as along the the longstanding $\Omega\left(n^{1.5}\right)$ barrier dating back to the early 70s [23, 31, 33]. Recently, Madry $[48,49]$ broke this barrier on unit-capacitated graphs, obtaining $\widetilde{O}\left(m^{10 / 7}\right)$ running time.

Our contribution. Building on recent work on iterative refinement for $p$-norm optimization [2], we develop a high accuracy algorithm for a large family of flow optimization problems on undirected graphs. For any $p \geq 2$, given weights $r \in \mathbb{R}_{\geq 0}^{E}$, a "gradient" $\boldsymbol{g} \in \mathbb{R}^{E}$, and a demand vector $\boldsymbol{b} \in \mathbb{R}^{V}$ (with $\boldsymbol{b}^{\top} \mathbf{1}=0$ ), we can solve

$$
\min _{B^{\top} f=b} \sum_{e} g_{e} f_{e}+r_{e} f_{e}^{2}+\left|f_{e}\right|^{p}
$$

to, $1 / \operatorname{poly}(n)$ additive error in time $2^{O\left(p^{3 / 2}\right)} m^{1+O\left(\frac{1}{\sqrt{p}}\right)}$ (Theorem 1.1). Using $\|f\|_{2, r}=\sqrt{\sum_{e} r_{e} f_{e}^{2}}$ to denote the $\boldsymbol{r}$-weighted 2-norm, the objective can also be viewed as $\boldsymbol{g}^{\top} \boldsymbol{f}+\|f\|_{2, \boldsymbol{r}}^{2}+\|f\|_{p}^{p}$.

Picking $\boldsymbol{g}, \boldsymbol{r}=\mathbf{0}$ gives us an $2^{O\left(p^{3 / 2}\right)} m^{1+O\left(\frac{1}{\sqrt{p}}\right)}$ time high-accuracy algorithm for $p$-norm minimizing flows on unit weighted undirected graphs $(p \geq 2)$. For large $p$, e.g. $p=\sqrt{\log n}$, this is an $m^{1+o(1)}$ time algorithm, and to our knowledge the first almost linear time highaccuracy algorithm for a flow problem other than Laplacian solvers $\left(\ell_{2}\right)$ or shortest-paths $\left(\ell_{1}\right)$.

A $p$-norm minimizing flow, for a large $p$, say $\sqrt{\log n}$, is trivially an $m^{o(1)}$ approximation to the maximum flow problem. Furthermore, we can leverage the combination of $\ell_{2}$ and $\ell_{p}$ norms in our problem (2) to design an almost-linear time low-width oracle that can be combined with multiplicative weights, as in the work of Christiano et al. [13], to obtain an almost-linear time lowaccuracy algorithm for maximum flow in unit capacity undirected graphs. This algorithm finds a $(1+\varepsilon)$-approximate maximum flow in $m^{1+o(1)}$ poly $(1 / \varepsilon)$ time. Using our Problem (2) solutions as a lowwidth oracle in multiplicative weights also gives us the first almostlinear time low accuracy algorithms to a problem known as isotropic total variation denoising $[57,68]$.

Based on dual solutions to Problem (2), we can get high-accuracy solutions to a semi-supervised learning problem on graphs in almost linear time. We discuss these problems and applications in more detail in Section 1.2.

Our methods. Instead of relying on solvers for linear systems, we show that algorithms inspired by fast solvers for systems of linear equations can allow us to directly solve non-linear problems to high accuracy. Two key features that make linear systems very special are: 1) Iterative refinement: the phenomenon that an algorithm that solves a linear system to a crude approximation, can be applied repeatedly to obtain a high-accuracy solution, and 2) preconditioning: the phenomenon that a solution to a carefully chosen, but much simpler, linear system gives a crude solution to the original harder-to-solve system. The powerful synergy between these two ingredients for linear systems, pioneered in the work of Spielman and Teng [66], forms the basis for nearly-linear time algorithms for solving several families of linear systems [15, 16, 19, 40,67].

There has been some success in adapting preconditioning to problems beyond linear systems, notably yielding nearly-linear time low-accuracy algorithms for undirected maximum flow [35, 54, 6163]. However, these algorithms need to employ a smoothing of the objective (e.g. switching from max to soft-max) in order to apply iterative methods such as gradient descent, or multiplicative weights, and there are no known high-accuracy fast algorithms for these smoothed objectives.

However, a recent result involving a subset of the authors [2] shows that high-accuracy iterative refinement is in fact a much more general phenomenon that applies to general $p$-norm optimization problems, for all $p$ that are bounded away from 1 and $\infty$. Building on the ideas in [2], we show that the class of smoothed $\ell_{p}$ norm minimization problems (Problem (2)) is closed under iterative refinement.

This opens up a major question: can we develop an appropriate notion of preconditioning, the other central ingredient of fast solvers for linear systems, applicable to smoothed $p$-norm problems? We resolve this question in the affirmative, and develop a theory of preconditioning that works for a wide class of non-linear problems. Before we describe our notion of preconditioning further, we note that the earlier works on preconditioning for non-linear (maximum) flow problems all relied on oblivious routing which gives rise to linear preconditioners. Oblivious preconditioners cannot possibly work well for accurate iterative refinement for $p$-norms. Consider changing a single coordinate from, say 1 , to $(1+\delta)$. If the update $\delta$ is much smaller than 1 in absolute value, the change in the objective from $1^{p}$ to $(1+\delta)^{p}$ is dominated by terms that scale as $\delta$ and $\delta^{2}$. However, if the update is much larger than 1 , the change is dominated by a $\delta^{p}$ term. This means that good preconditioning across small and large updates is inherently highly dependent on the current coordinate value, and hence cannot be oblivious.

Our approach to preconditioning allows us to simultaneously control terms of different degrees (namely scaling as $\delta, \delta^{2}$, and $\delta^{p}$ ) for flow problems on graphs. We develop sparsification that preserves these three different types of objectives, in an way that crucially depends on the current solution that we wish to update. As identified above, this adaptive preconditioning is necessary for the non-linear problems we consider. We also show that unit-weighted graphs have "multi-objective low-stretch trees". Combining these trees with the sparsification algorithms we develop, we obtain algorithms for ultra-sparsification for our class of non-linear problems.

Finally, we can now interleave the non-linear iterative-refinement approach developed in [2], with the notion of non-linear preconditioning we develop, to obtain an algorithm in the spirit of the original Laplacian solver of Spielman and Teng, that obtains a highaccuracy solution for our non-linear flow problems (Problem (2)) in almost-linear time.

Discussion. We believe that the overall algorithmic approach established here, namely the use of non-linear iterative refinement and preconditioning together, may potentially be applicable far beyond the class of problems studied in this paper. In particular, we hope that our techniques can be refined to improve the dependence on $p$, and potentially lead to better algorithms for larger $p$, or even $p=\infty$, implying better algorithms for (approximate) max-flow.

We also believe that by developing a more complete theory of elimination and sparsification for these objectives, it will be possible 
to sparsify non-unit weight graphs, and develop solvers following the patterns of non-tree based Laplacian solvers [42, 43, 55].

\subsection{Related Work}

The two works most directly related to our results are - the work on iterative refinement for $\ell_{p}$-norms by Adil et al. [2], and the recursive-preconditioning framework of the Spielman-Teng Laplacian solver [67]. Given our iterative refinement procedure for smoothed $p$-norm problems, our goal is to develop matching preconditioning schemes to obtain fast solvers. Many conceptual obstacles arise in trying to realize this vision, preventing us from adopting subsequent fast solvers for Laplacian linear systems that have greatly simplified the result of Spielman and Teng.

We require low-stretch trees $[1,7]$ to build our preconditioners. A low-stretch tree is sufficient to construct a nearly-linear time Laplacian solver [36, 44]. These solvers compute a single low-stretch tree, and then make an almost-linear number of low-cost updates to a solution, as determined by the tree. However, our tree needs to change every time our current solution changes, and hence this approach cannot work for us. We cannot yet implement solvers based on global matrix concentration arguments [38, 42, 43], since no such concentration theory currently exists for the smoothed p-norm objectives we consider.

Our approach to build adaptive preconditioners still draws on ideas from oblivious routing, and has much in common with the construction of oblivious routings and cut approximators using low-stretch trees from [56] and J-trees [35, 47, 61]. For graph sparsification, our methods diverge substantially from Spielman-Teng, since our sparsification procedure needs to preserve the gradient terms, and the $\ell_{p}$-norm terms, in addition to the $\ell_{2}$-norm terms in the smoothed $p$-norm objective.

The ability of our sparsifier and associated flow-mappings to preserve linear terms in the objective exactly is related to the "sparsification with additional constraints" that is present in the Eulerian sparsification of $[14,16]$.

We are able to build preconditioners only when all the $p$-power terms have unit weights. Hence, we need to construct unweighted sparsifiers. This is related to the unit weight sparsifiers by Anderson, Gu and Melgaard [8] and improved by Liao, Orecchia, and AllenZhu'15 [6, Appendix C].

The routing arguments we use to construct maps between a graph and its sparsifier that simultaneously preserve 2-norm and $p$-norm objectives are related to the flow-sparsifier construction from [35].

Our sparsification algorithm is based on the approach from Spielman-Teng [67], that relies on expander decompositions. Importantly, our approach requires stronger expander decomposition results proved recently [58]. Additionally, a uniformity condition we enforce on the gradient to control the cycle-space sampling errors is related to Lewis weight-based sampling studied in [18].

We expect that our algorithm can be greatly simplified and adapted to non-unit weighted graphs if sufficient sampling concentration theory is developed for the objectives we study, and that this may be possible using Lewis-weight-like techniques. Unfortunately, the methods of [18] rely on fairly intricate proofs, and need to be generalized to our smoothed $p$-norm objectives.
The development of high-accuracy algorithms for $p$-norm minimization that are faster than interior point methods (IPMs) [50] was pioneered by the recent work of Bubeck et al. [11] which introduced $\gamma$-functions that were also used in [2]. However, the role played by these functions in [11] is very different than in [2] and our work. The methods in [11] are conceptually similar to interior point methods (IPMs) [50] (as in they are homotopy methods). Their runtime for large $p$ behaves essentially like IPMs, requiring about $m^{3 / 2-o(1)}$ time for solving $p$-norm flow problems, giving little improvement over the $\mathrm{m}^{3 / 2+o(1)}$ time required by IPMs. The approach of [2] is fundamentally different, in that, it gives an iterative refinement method that achieves $m^{4 / 3-o(1)}$ running time for large $p$. Our algorithm achieves $m^{1+o(1)}$ running time for large $p$, and relies on the iterative-refinement techniques developed in [2].

Another very interesting line of work on fast solvers for nonlinear flow problems is the work on matrix scaling [5, 17]. Algorithms that achieve nearly linear running time for this problem depend on the condition number $\kappa$ of the problem (which may be exponentially large), and their running time scales as $\log \kappa$. In contrast, standard high-accuracy convex programming techniques (IPMs and Ellipsoid method) achieve a $\log \log \kappa$ dependence in the running time for this problem, so the nearly linear time algorithms are not comparable to conventional high-accuracy algorithms.

\subsection{Main Results and Applications}

Our main algorithm solves the following problem to high accuracy.

$$
\min _{B^{\top} f=b} \sum_{e} g_{e} f_{e}+r_{e} f_{e}^{2}+\left|f_{e}\right|^{p}
$$

Let $\operatorname{val}(f)$ denote value of a flow $f$ according to the above objective, and let OPT denote value of the optimal solution to Problem (2). Our main result is the following statement which is a corollary of our main technical theorem in Section 4.

TheOREM 1.1 (SMOothed $p$-NORM Flows). For any $p \geq 2$, given weights $\boldsymbol{r} \in \mathbb{R}_{\geq 0}^{E}$, a "gradient" $\boldsymbol{g} \in \mathbb{R}^{E}$, a demand vector $\boldsymbol{b} \in \mathbb{R}^{V}$ (with $\left.\boldsymbol{b}^{\top} \mathbf{1}=0\right)$, and an initial solution $f^{(0)}$ such that all parameters are bounded by $2^{\text {poly }(\log n)}$, we can compute a flow $\widetilde{f}$ satisfying demands $b$, i.e., $B^{G \top} \widetilde{f}=b$, such that

$$
\operatorname{val}(\tilde{f})-O P T \leq \frac{1}{\operatorname{poly}(m)}\left(\operatorname{val}\left(f^{(0)}\right)-O P T\right)+\frac{1}{\operatorname{poly}(m)}
$$

in $2^{O\left(p^{3 / 2}\right)} m^{1+O\left(\frac{1}{\sqrt{p}}\right)}$ time, where $m$ is the number of edges in $G$.

From this, we also get a (slightly simpler) statement about $p$ norm flows.

TheOrem 1.2 ( $p$-Norm Flows). For any $p \geq 2$, unweighted graph $G(V, E)$ and demands $\boldsymbol{b}$, there exists a routine $\operatorname{PFLOWs}(\mathcal{G}, \boldsymbol{b})$ we can compute a flow $\widetilde{f}$ satisfying satisfying $\boldsymbol{b}$, i.e., $B^{G \top} \widetilde{f}=\boldsymbol{b}$, such that

$$
\|\widetilde{f}\|_{p}^{p} \leq\left(1+\frac{1}{p o l y(m)}\right) \min _{f: B^{G \top} f=b}\|f\|_{p}^{p} .
$$

in $2^{O\left(p^{3 / 2}\right)} m^{1+O\left(\frac{1}{\sqrt{p}}\right)}$, time, where $m$ is the number of edges in $G$.

The details of the above two theorems are in the full version of the paper. 
Our algorithm has direct applications to problems from machine learning, combinatorial optimization, and image processing.

$\ell_{p}$-norm Semi-Supervised Learning on Graphs. Semi-supervised learning on graphs in machine learning is often based on solving an optimization problem where voltages (labels) are fixed at some vertices in a graph the voltages at remaning nodes are chosen so that some overall objective is minimized (e.g. $p$-norm of the vector of voltage differences across edges) [4, 22, 41]. Formally, given a graph $G=(V, E)$ and a labelled subset of the nodes $T \subset V$ with labels $\boldsymbol{s}_{T} \in \mathbb{R}^{T}$, we can write the problem as

$$
\min _{x \in \mathfrak{R}^{V} \mid x_{T}=s_{T}} \sum_{u \sim v}\left|x_{u}-x_{v}\right|^{p} .
$$

By converting this problem to its dual, we get an almost linear time algorithm for solving it to high accuracy, provided the initial voltage problem uses $p$ close to 1 : In this case, voltage solutions are "cutlike". Given $p<2$, we get a solver that computes a $(1+1 / \operatorname{poly}(m))$ multiplicative accuracy solution in time $2^{O\left(\left(\frac{1}{p-1}\right)^{3 / 2}\right)} m^{1+O(\sqrt{p-1})}$. For $p=1+\frac{1}{\sqrt{\log n}}$, this is time is bounded by $m^{1+o(1)}$.

Converting the dual of Problem (3) into a form solvable by our algorithms requires a small transformation, which we leave to the full version.

$p$-norm Flow Oracles. The mixed $\ell_{2}^{2}$ and $\ell_{p}^{p}$ objective in our Problem (2) is useful for building oracles to use in multiplicative weight update algorithms based on flows, as they appear in $[2,13]$. Assume we are looking to solve some problem to $(1+\epsilon)$-accuracy measured as multiplicative error, and let us assume $\frac{1}{\operatorname{poly}(m)}<\epsilon<0.5$. Specifically we can solve for the following objective subject to certain linear constraints.

$$
\sum_{e} \boldsymbol{r}_{e} f_{e}^{2}+\frac{\epsilon\|\boldsymbol{r}\|_{1}}{m}\left|f_{e}\right|^{p}
$$

This gives an oracle for several problems. Algorithms based on oracle solutions to this type of objective work by noting that any $f$ with $\|f\|_{\infty} \leq 1$ gives an objective value at most

$$
\sum_{e} \boldsymbol{r}_{e} f_{e}^{2}+\frac{\epsilon\|\boldsymbol{r}\|_{1}}{m}\left|f_{e}\right|^{p} \leq(1+\epsilon)\|\boldsymbol{r}\|_{1} .
$$

Since such a flow must exist in the context where the oracle is applied, the optimum flow must also meet this bound. Now, if we compute a $(1+0.01 \epsilon)$ approximately optimal solution to this problem, it must satisfy

$$
\sum_{e} \boldsymbol{r}_{e} f_{e}^{2}+\frac{\epsilon\|\boldsymbol{r}\|_{1}}{m}\left|f_{e}\right|^{p} \leq(1+1.1 \epsilon)\|\boldsymbol{r}\|_{1} .
$$

By Cauchy-Schwarz, we get $\sum_{e} \boldsymbol{r}_{e}\left|f_{e}\right| \leq \sqrt{\|\boldsymbol{r}\|_{1} \sum_{e} \boldsymbol{r}_{e} f_{e}^{2}} \leq(1+1.1 \epsilon) \| \boldsymbol{r}$ which tells us the oracle is "good-on-average" according to the weights $r$. The objective value also implies for every edge that

$$
\frac{\epsilon\|\boldsymbol{r}\|_{1}}{m}\left|f_{e}\right|^{p} \leq(1+1.1 \epsilon)\|\boldsymbol{r}\|_{1} \leq 2\|\boldsymbol{r}\|_{1},
$$

which simplifies to: $\left|f_{e}\right| \leq(m / \epsilon)^{1 / p} \leq m^{o(1)}$, when we set $p=$ $\log ^{0.1} n$. This is the width of the oracle, and together these conditions demonstrate that the oracle suffices for a multiplicative weights algorithm and bounds the number of calls to the oracle by $m^{o(1)} \operatorname{poly}(1 / \epsilon)$.
This oracle has multiple applications:

Approximate undirected maximum flow:: Using the oracle, we can approximate maximum flow using [13], giving an algorithm for undirected maximum flow that is not based on oblivious routings unlike other fast algorithms for approximate maximum flow [35, $54,61]$. Our algorithm obtains almost-linear time, albeit only for unit weighted graphs.

Isotropic total variation denoising.: Using our algorithm, we can give the first almost linear time, low accuracy algorithm for total variation denoising on unit weighted graphs $[57,68]$. While there has been significant advances in image processing since the introduction of this objective, it still remains a representative objective in pixel vision tasks. The total variation objectives can be viewed as variants of semi-supervised learning on graphs: Given a "signal" vector $s$ which corresponds to noisy observations of pixels of an image, we want to find a denoised version of $s$, which we refer to as $x$. The denoised output $x$ should minimize an objective that measures both the between pixels in $x$ that are close to each other in the image (which should be small), and the difference between $x$ and $s$ (which should also be small). The most popular version of this problem, known as isotropic total variation denoising, allows the input to specify a collection of groups of pixels with connections inside each group $i$ given by a set of edges $E_{i}$, and asks that 1 ) the denoised pixels are close in an $\ell_{2}$ sense to the measured signal, 2) in each group, the standard deviation between denoised pixels is not too high. These goals are expressed in the objective

$$
\sum_{u}\left(x_{u}-s_{u}\right)^{2}+\sum_{i} \sqrt{\sum_{e \in E_{i}}\left(x_{u}-x_{v}\right)^{2}} .
$$

The dual of this problem is grouped flows, which is finding $f$ such that $\boldsymbol{B}^{\top} \boldsymbol{f}=\boldsymbol{d}$ and for edge sets $E_{i}$,

$$
\left\|f_{E_{i}}\right\|_{2}^{2} \leq 1
$$

Our oracle gives the first routine for approximate isotropic $T V$ denoising that runs in almost linear time. The previous best running time was about $m^{4 / 3}$ [12].

\section{OVERVIEW}

Iterative refinement and closure under refinement. Suppose we want to update some tentative solution $f$ to the minimization problem

$$
\min _{B^{\top} f=b}\|f\|_{p}^{p}
$$

by stepping from $f$ to $f+\delta$, and hopefully improve the objective value substantially. [2] showed that we can define an update problem with the property that if we find even a crude approximate minimizer $\widetilde{\boldsymbol{\delta}}$ of this update problem, we can move to a new point $f^{\prime}=f+\widetilde{\delta}$, so that the gap to the optimum in the original optimization problem (4) will decrease by a constant factor (depending only on $p)$ from $\|f\|_{p}^{p}$ - OPT to $\left\|f^{\prime}\right\|_{p}^{p}-\mathrm{OPT} \leq\left(1-2^{-O(p)}\right)\left(\|f\|_{p}^{p}-\mathrm{OPT}\right)$. In other words, we have a kind of iterative refinement: crude solutions to an update problem directly give constant factor progress in the original objective.

Note that $\|f\|_{p}^{p}=\sum_{i}\left|f_{i}\right|^{p}$. This will help us understand the objective function of the update problem coordinate-wise. Our update problem objective function is motivated by the following 
observations. Our function differs slightly from the function used in [2], which in turn was based on functions from [11]. Suppose $p \geq 2$ is an even integer (only to avoid writing absolute values), then

$$
\begin{aligned}
& f_{i}^{p}+p f_{i}^{p-1} \delta_{i}+2^{-O(p)} \underbrace{\left(f_{i}^{p-2} \delta_{i}^{2}+\delta_{i}^{p}\right)}_{\text {write as } h_{p}\left(f_{i}^{p-2}, \delta_{i}\right)} \\
& \leq\left(f_{i}+\delta_{i}\right)^{p} \\
& \leq f_{i}^{p}+p f_{i}^{p-1} \delta_{i}+2^{O(p)} \underbrace{\left.f_{i}^{p-2} \delta_{i}^{2}+\delta_{i}^{p}\right)}_{h_{p}\left(f_{i}^{p-2}, \delta_{i}\right)}
\end{aligned}
$$

Of course, the exact expansion gives

$\left(f_{i}+\delta_{i}\right)^{p}=$

$f_{i}^{p}+p f_{i}^{p-1} \delta_{i}+\frac{p(p-1)}{2} f_{i}^{p-2} \delta_{i}^{2}+\frac{p(p-1)(p-2)}{6} f_{i}^{p-3} \delta_{i}^{3}+\ldots+\delta_{i}^{p}$

So essentially we can approximate this expansion using only the zeroth, first, second, and last term in the expansion. We use $g(f)$ to denote the vector with $g_{i}(f)=p f_{i}^{p-1}$ (i.e. the gradient), and let $f^{p-2}$ denote the entrywise powered vector, and define $h_{p}\left(f^{p-2}, \delta\right)=$ $\sum_{i} h_{p}\left(f_{i}^{p-2}, \boldsymbol{\delta}_{i}\right)$. Thus we have

$2^{-O(p)} h_{p}\left(f^{p-2}, \boldsymbol{\delta}\right) \leq\|f+\boldsymbol{\delta}\|_{p}^{p}-\|\boldsymbol{f}\|_{p}^{p}-\boldsymbol{g}(\boldsymbol{f})^{\top} \boldsymbol{\delta} \leq 2^{O(p)} h_{p}\left(f^{p-2}, \boldsymbol{\delta}\right)$

Note that for any scalar $0<\lambda<1$,

$$
\lambda^{p} h_{p}\left(f^{p-2}, \boldsymbol{\delta}\right) \leq h_{p}\left(f^{p-2}, \lambda \boldsymbol{\delta}\right) \leq \lambda^{2} h_{p}\left(f^{p-2}, \boldsymbol{\delta}\right) .
$$

Together, these observations are enough to ensure that if we have $\widetilde{\delta}$ which is a constant factor approximate solution to the following optimization problem, which we define as our update problem

$$
\min _{\boldsymbol{B}^{\top} \boldsymbol{\delta}=\mathbf{0}} \boldsymbol{g}(\boldsymbol{f})^{\top} \boldsymbol{\delta}+h_{p}\left(f^{p-2}, \boldsymbol{\delta}\right)
$$

then we can find a $\lambda$ s.t.

$$
\|f+\lambda \widetilde{\boldsymbol{\delta}}\|_{p}^{p}-\mathrm{OPT} \leq\left(1-2^{-O(p)}\right)\left(\|f\|_{p}^{p}-\mathrm{OPT}\right) .
$$

Note that Problem (6) is an instance of Problem (2).

But what have we gained? Why is Problem (6) more tractable than the one we started with? A key reason is that unlike the exact expansion of an update as given by Equation (5), all the higher order terms in the objective function of (6) are coordinate-wise even functions, i.e. flipping the sign of a coordinate does not change the value of the function. [2] used a different but still even function instead of our $h_{p}$. This symmetrization allowed them to develop a multiplicative weights update algorithm motivated by [13] for their version of Problem (6), reducing the problem to solving a sequence of linear equations.

For our choice of $h_{p}$, it is particularly simple to show another very important property: Consider solving Problem (6) by again applying iterative refinement to this problem. That is, at some intermediate step with $\delta$ being the current solution, we aim to find an update $\widehat{\boldsymbol{\delta}}$ s.t. $\boldsymbol{B}^{\top} \widehat{\boldsymbol{\delta}}=\mathbf{0}$ and $\boldsymbol{g}(f)^{\top}(\boldsymbol{\delta}+\widehat{\boldsymbol{\delta}})+h_{p}\left(f^{p-2}, \boldsymbol{\delta}+\widehat{\boldsymbol{\delta}}\right)$ is smaller than $\boldsymbol{g}(\boldsymbol{f})^{\top}(\boldsymbol{\delta})+h_{p}\left(f^{p-2}, \boldsymbol{\delta}\right)$. Then by expanding the two non-linear terms of $h_{p}\left(f_{i}^{p-2}, \boldsymbol{\delta}_{i}\right)$, i.e. $\left(\boldsymbol{\delta}_{i}+\widehat{\boldsymbol{\delta}}\right)^{p}$ and $\left(\boldsymbol{\delta}_{i}+\widehat{\boldsymbol{\delta}}^{2}\right.$, similar to Equation (5), we get a sequence of terms with powers of $\boldsymbol{\delta}_{i}$ ranging from 2 to $p$. If we approximate this sequence again using only the $\widehat{\boldsymbol{\delta}}_{i}^{2}$ and $\widehat{\boldsymbol{\delta}}_{i}^{p}$ terms, we get a new update problem that is another instance of Problem (2). In general, we can set up iterative refinement update problems for instances of Problem (2), and get back another problem of the same class (after our approximation based on dropping intermediate terms). Thus, the problem class (2) is closed under repeatedly creating iterative update problems. This observation is central because it allows us to develop recursive algorithms.

Adaptive preconditioning. Earlier work on preconditioning for non-linear (maximum) flow problems all relied on oblivious routing which gives rise to linear preconditioners. These inherently cannot work well for high-accuracy iterative refinement, and the issue is not merely linearity: Consider Problem (6): the optimal $\boldsymbol{\delta}$ is highly dependent on the current $f$, and when a coordinate $\boldsymbol{\delta}_{i}$ is large compared to the current $\left|f_{i}\right|$, the function depends on it as $\delta_{i}^{p}$, while when $\delta_{i}$ is small compared to $\left|f_{i}\right|$, it behaves as $\delta_{i}^{2}$. Thus the behavior is highly dependent on the current solution. This necessitates adaptive and non-linear preconditioners.

To develop adaptive preconditioners, we employ recursive chains of alternating calls to non-linear iterative refinement and a new type of (ultra-)sparsification that is more general and stronger, allowing us to simultanously preserve multiple different properties of our problem. And crucially, every time our solution is updated, our preconditioners change. Following the template of the SpielmanTeng Laplacian solver, we recursively solve a problem on $m$ edges by solving about $\kappa$ problems on graphs with $n-1+m / \kappa$ edges. These ultra-sparse graphs allow us to eliminate degree 1 and 2 vertices and obtain a smaller problem. Because our update problem (Problem (6)) corresponds to a flow-circulation problem with some objective, we are able to understand elimination on these objectives in a relatively simple way: the flow on degree 1 and 2 vertices is easily related to flow in a smaller graph created by elimination. Unlike SpielmanTeng, every recursive call must rely on a new graph sparsifier, because the "graph" we sparsify depends heavily on the current solution that we are seeking to update: we have to simultaneously preserve linear, quadratic and $p$-th order terms, whose weights depend on the current solution. We also need maps back and forth between the original graph and the new graph so a good solution on a smaller graph can be transformed into a good solution on the larger graph ${ }^{1}$.

There are several components to building the ultra-sparse graphs that allow us to create preconditioners using iterative refinement and elimination. Overall, our approach to creating smaller instances is based on finding a subtree of the current graph, and moving most edges onto a small subset of the vertices by re-routing them along the tree. This ensures that many vertices are degree 1 or 2 and can be eliminated, resulting in a graph on much fewer vertices. We can keep this graph reasonably sparse by sparsifying its edges. We discuss these components of the ultra-sparsication procedure in more detail next.

Tree-routing to get graphs on few vertices. Given a potentially dense graph, we wish to move most edges onto a few vertices of a

${ }^{1}$ Only the map from the smaller graph to the larger has to be constructive, although both are in our case. 
tree, so that many of the remaining vertices are low degree and can be eliminated. Our high-level approach is the same as SpielmanTeng [67], where we utilize a low-stretch spanning tree, and move off-tree edges to a small set of portal nodes. However, when we re-route flow on the graph where most edges are moved using the tree to be between just a few vertices, we need to (approximately) preserve three different properties of the flow on the original graph, namely the inner product between gradients and flows $\sum_{e} g_{e} f_{e}$, the 2-norm energy $\sum_{e} r_{e} f_{e}^{2}$, and the $p$-norm energy $\sum_{e} f_{e}^{p}$. It turns out that we can move edges around on our graphs to produce a new graph while exactly preserving the linear term $\sum_{e} g_{e} f_{e}$ for flows mapped between one graph and the other. This means any tree is acceptable from the point of preserving the linear term. To move edges around and bound distortion of solutions w.r.t. the quadratic $\boldsymbol{r}_{e} f_{e}^{2}$ term, we use a low stretch tree w.r.t. the $\boldsymbol{r}$ weights as resistances. This leaves us with little flexibility for the $\sum_{e} f_{e}^{p}$ term. However, for large $p$, provided every $p$-th order term is weighted the same (i.e. we have $s \sum_{e} f_{e}^{p}$ instead of $\sum_{e} s_{e} f_{e}^{p}$ ), it turns out that, moving edges along any tree will result in bounded distortion of the solution, provided we are careful about how we move those edges. Thus, we can move edges around carefully to be among a small subset of the portal nodes while simultaneously controlling all linear, 2-nd order and $p$-th order terms. But, this only works if all the $p$-th order terms are weighted the same. This leads us to maintain uniform-weighted $p$-th order terms as an invariant throughout the algorithm. The iterative refinement steps naturally weigh all $p$-th order terms the same provided the original function does. However, our sparsification procedures do not immediately achieve this, but we show we can enforce this uniform-weight invariant with only a manageable additional distortion of our solutions. Elimination also does not naturally weigh all $p$-th order terms the same even in our case when the original function does, but we can bound the distortion incurred by explicitly making the weights uniform. Our tree-based edge re-routing naturally creates maps between solutions on the old and new graphs.

Graph sparsification and solution mapping. Once we are able to move most of the edges onto a small subset of vertices, we wish to sparsify the resulting dense graph over those vertices. This sparsification has to simultaneously preserve properties of 1-st, 2-nd and $p$-th order terms, as well as the interactions between them, which turns out to be challenging. We resort to expander decomposition which allows us to partition the vertex set such that the edges internal to each subset form an expander and not too many edges cross the partitions. Just having an expander graph is not enough to allow us to sample the graph due to the need of preserving the linear terms. Thus, we also require that on each expander the orthogonal projection of the gradient to the cycle space of the sub-graph has its maximum squared entry not much larger than the the average squared entry. We refer to this as a uniform (projected) gradient. We discuss how to obtain an expander decomposition that guarantees the projected gradients are uniform in the expanders later in this overview. Given the uniform projected gradient condition, we show that we can uniformly sample edges of these expanders to create sparsified versions of them. We construct maps between the flows on an original expander and its sampled version that work for any flow, not only a circulation. These maps preserve the linear term $\sum_{e} \boldsymbol{g}_{e} f_{e}$ exactly, while bounding the cost of the 2-norm and $p$-norm terms by relating them to the cost of the optimal routing of a flow with the same demands and same gradient inner product, and showing that optimal solutions are similar on the original expander and its sampled version. This strategy resembles the flow maps developed in [35], and like their maps, we route demands using electrical flows on individual expanders, but additionally we need create a flow in the cycle space that depends on projection of the gradient onto the cycle space.

Expander decomposition. As mentioned above, we need to decompose a graph into expanders with the uniform projected gradient guarantee on each expander, i.e. on each expander, the orthogonal projection of the gradient to the cycle space of the induced graph has its maximum squared entry not much larger than the average squared entry. For our sampling guarantees it also becomes important that a recent work [58], involving one of the authors of this paper, makes it possible to compute expander decompositions in nearly linear time, where we can guarantee that each induced subgraph truly forms an expander. In contrast, all earlier fast expander decompositions produced weaker guarantees of each induced subgraph being "inside" a larger expander using other graph edges. With the weaker guarantee, it is unclear how to sample in a way that preserves interactions between the gradient term and other terms, because our algorithms achieve this by crucially relying on the projection of the gradient into the cycle and flow spaces of a sub-graph that must be an expander. We show that we can construct expanders with uniform projected gradients by recursively applying [58] to subgraphs that lack gradient uniformity, and this process does not cut too many edges so most edges are inside the expanders. In particular, if gradients are not uniform, the 2-norm of their projection in the cycle space must decay geometrically. This leads to a decomposition where gradients are uniform or have extremely small cycle-space projection, in which case we can afford to round them to zero.

Putting together the ultra-sparsifier. We need to show that adding together our individual sparsifiers results in a sparsifier of the overall graph. This is fairly immediate given the strong guarantees we established on the individual graphs. We also need to be able to repeatedly decompose and sparsify enough times that the overall graph becomes sparse. To address this issue, we use ideas from [38] that suggest scaling up the tree from the tree routing section limits the error incurred during sampling. Here it again becomes important that because we rely on [58], we know exactly which edges belong to a sparsifier. This guarantee limits the interaction between sparsification of different expanders.

\section{PRELIMINARIES}

\subsection{Smoothed $p$-norm functions}

We consider $p$-norms smoothed by the addition of a quadratic term. First we define such a smoothed $p$-power on $\mathbb{R}$.

Definition 3.1 (Smoothed p-power). Given $r, x \in \mathbb{R}, r \geq 0$ define the $r$-smoothed $s$-weighted $p$-power of $x$ to be

$$
h_{p}(r, s, x)=r x^{2}+s|x|^{p} \text {. }
$$


This definition can be naturally extended to vectors to obtained smoothed $p$-norms.

Definition 3.2 (Smoothed p-norm). Given vectors $x \in \mathbb{R}^{m}, \boldsymbol{r} \in$ $\mathbb{R}_{\geq 0}^{m}$, and a positive scalar $s \in \mathbb{R}_{\geq 0}$, define the $\boldsymbol{r}$-smooth $s$-weighted $p$-norm of $x$ to be

$$
h_{p}(\boldsymbol{r}, s, \boldsymbol{x})=\sum_{i=1}^{m} h_{p}\left(\boldsymbol{r}_{i}, s, \boldsymbol{x}_{i}\right)=\sum_{i=1}^{m}\left(\boldsymbol{r}_{i} x_{i}^{2}+s\left|x_{i}\right|^{p}\right) .
$$

Note that the coefficient of all the $p$-power term is the same.

\subsection{Flow Problems and Approximation}

We will consider problems where we seek to find flows minimizing smoothed $p$-norms. We first define these problem instances.

Definition 3.3 (Smoothed p-norm instance). A smoothed p-norm instance is a tuple $\mathcal{G}$,

$$
\mathcal{G} \stackrel{\text { def }}{=}\left(V^{\mathcal{G}}, E^{\mathcal{G}}, \boldsymbol{g}^{\mathcal{G}}, \boldsymbol{r}^{\mathcal{G}}, s^{\mathcal{G}}\right),
$$

where $V^{\mathcal{G}}$ is a set of vertices, $E^{\mathcal{G}}$ is a set of undirected edges on $V^{\mathcal{G}}$, the edges are accompanied by a gradient, specified by $\mathbf{g}^{\mathcal{G}} \in \mathbb{R}^{E^{\mathcal{G}}}$, the edges have $\ell_{2}^{2}$-resistances given by $\boldsymbol{r}^{\mathcal{G}} \in \mathbb{R}_{\geq 0}^{E^{\mathcal{G}}}$, and $s \mathcal{G} \in \mathbb{R}_{\geq 0}$ gives the $p$-norm scaling.

Definition 3.4 (Flows, residues, and circulations). Given a smoothed $p$-norm instance $\mathcal{G}$, a vector $f \in \mathbb{R}^{E^{\mathcal{G}}}$ is said to be a flow on $\mathcal{G}$. A flow vector $f$ satisfies residues $b \in \mathbb{R}^{V^{\mathcal{G}}}$ if $\left(B^{\mathcal{G}}\right)^{\top} f=b$, where $B^{\mathcal{G}} \in \mathbb{R}^{E^{\mathcal{G}} \times V^{\mathcal{G}}}$ is the edge-vertex incidence matrix of the graph $\left(V^{\mathcal{G}}, E^{\mathcal{G}}\right)$, i.e., $\left(B^{\mathcal{G}}\right)_{(u, v)}^{\top}=\mathbf{1}_{u}-\mathbf{1}_{v}$.

A flow $f$ with residue 0 is called a circulation on $\mathcal{G}$.

Note that our underlying instance and the edges are undirected. However, for every undirected edge $e=(u, v) \in E$, we assign an arbitrary fixed direction to the edge, say $u \rightarrow v$, and interpret $f_{e} \geq 0$ as flow in the direction of the edge from $u$ to $v$, and $f_{e}<0$ as flow in the reverse direction. For convenience, we assume that for any edge $(u, v) \in E$, we have $f_{(u, v)}=-f_{(v, u)}$.

Definition 3.5 (Objective, $\mathcal{E}^{\mathcal{G}}$ ). Given a smoothed p-norm instance $\mathcal{G}$, and a flow $f$ on $\mathcal{G}$, the associated objective function, or the energy, of $f$ is given by

$$
\mathcal{E}^{\mathcal{G}}(f)=\left(\boldsymbol{g}^{\mathcal{G}}\right)^{\top} f-h_{p}(\boldsymbol{r}, s, f) .
$$

Definition 3.6 (Smoothedp-norm flow/ circulation problem). Given a smoothed $p$-norm instance $\mathcal{G}$ and a residue vector $\boldsymbol{b} \in \mathbb{R}^{E^{\mathcal{G}}}$, the smoothed p-norm flow problem $(\mathcal{G}, \boldsymbol{b})$, finds a flow $f \in \mathbb{R}^{E^{\mathcal{G}}}$ with residues $\boldsymbol{b}$ that maximizes $\mathcal{E}^{\mathcal{G}}(f)$, i.e.,

$$
\max _{f:\left(B^{\mathcal{G}}\right)^{\top} f=b} \mathcal{E}^{\mathcal{G}}(f) .
$$

If $\boldsymbol{b}=\mathbf{0}$, we call it a smoothed p-norm circulation problem.

Note that the optimal objective of a smoothed $p$-norm circulation problem is always non-negative, whereas for a smoothed $p$-norm flow problem, it could be negative.

\subsection{Approximating smoothed $p$-norm instances}

Since we work with objective functions that are non-standard (and not even homogeneous), we need to carefully define a new notion of approximation for these instances.

Definition $3.7\left(\mathcal{H} \leq_{\kappa} \mathcal{G}\right)$. For two smoothed $p$-norm instances, $\mathcal{G}, \mathcal{H}$, we write $\mathcal{H} \leq_{\kappa} \mathcal{G}$ if there is a linear map $\mathcal{M}_{\mathcal{H} \rightarrow \mathcal{G}}: \mathbb{R}^{E^{\mathcal{H}}} \rightarrow$ $\mathbb{R}^{E^{\mathcal{G}}}$ such that for every flow $f^{\mathcal{H}}$ on $\mathcal{H}, f^{\mathcal{G}}=\mathcal{M}_{\mathcal{H} \rightarrow \mathcal{G}}\left(f^{\mathcal{H}}\right)$ is a flow on $\mathcal{G}$ such that

(1) $f^{\mathcal{G}}$ has the same residues as $f^{\mathcal{H}}$ i.e., $\left(B^{\mathcal{G}}\right)^{\top} f^{\mathcal{G}}=\left(B^{\mathcal{H}}\right)^{\top} f^{\mathcal{H}}$, and

(2) has energy bounded by:

$$
\frac{1}{\kappa} \mathcal{E}^{\mathcal{H}}\left(f^{\mathcal{H}}\right) \leq \mathcal{E}^{\mathcal{G}}\left(\frac{1}{\kappa} f^{\mathcal{G}}\right)
$$

For some of our transformations on graphs, we will be able to prove approximation guarantees only for circulations. Thus, we define the following notion restricted to circulations.

Definition $3.8\left(\mathcal{H} \subseteq_{\kappa}^{c y c l e} \mathcal{G}\right.$ ). For two smoothed $p$-norm instances, $\mathcal{G}, \mathcal{H}$, we write $\mathcal{H} \coprod_{\kappa}^{\text {cycle }} \mathcal{G}$ if there is a linear map $\mathcal{M}_{\mathcal{H} \rightarrow \mathcal{G}}$ : $\mathbb{R}^{E^{\mathcal{H}}} \rightarrow \mathbb{R}^{E^{\mathcal{G}}}$ such that for any circulation $f^{\mathcal{H}}$ on $\mathcal{H}$, i.e., $\left(B^{\mathcal{H}}\right)^{\top} f^{\mathcal{H}}=$ 0, the flow $f^{\mathcal{G}}=\mathcal{M}_{\mathcal{H} \rightarrow \mathcal{G}}\left(f^{\mathcal{H}}\right)$ is a circulation, i.e., $\left(B^{\mathcal{G}}\right)^{\top} f^{\mathcal{G}}=\mathbf{0}$, and satisfies

$$
\frac{1}{\kappa} \mathcal{E}^{\mathcal{H}}\left(f^{\mathcal{H}}\right) \leq \mathcal{E}^{\mathcal{G}}\left(\frac{1}{\kappa} f^{\mathcal{G}}\right)
$$

Observe that $\mathcal{H} \leq_{\kappa} \mathcal{G}$ implies $\mathcal{H} \preceq_{\kappa}^{\text {cycle }} \mathcal{G}$.

These definitions satisfy most properties that we want from comparisons.

Lemma 3.9 (Reflexivity). For every smoothed p-norm instance $\mathcal{G}$, and every $\kappa \geq 1, \mathcal{G} \leq_{\kappa} \mathcal{G}$ and $\mathcal{G} \leq_{\kappa}^{\text {cycle }} \mathcal{G}$ with the identity map.

It behaves well under composition.

Lemma 3.10 (Composition). Given two smoothed p-norm instances, $\mathcal{G}_{1}, \mathcal{G}_{2}$, such that $\mathcal{G}_{1} \unlhd_{\kappa_{1}} \mathcal{G}_{2}$ with the map $\mathcal{M}_{\mathcal{G}_{1} \rightarrow \mathcal{G}_{2}}$ and $\mathcal{G}_{2} \unlhd_{\kappa_{2}} \mathcal{G}_{3}$ with the map $\mathcal{M}_{\mathcal{G}_{2} \rightarrow \mathcal{G}_{3}}$, then $\mathcal{G}_{1} \unlhd_{\kappa_{1} \kappa_{2}} \mathcal{G}_{3}$ with the map $\mathcal{M}_{\mathcal{G}_{1} \rightarrow \mathcal{G}_{3}}=\mathcal{M}_{\mathcal{G}_{2} \rightarrow \mathcal{G}_{3}} \circ \mathcal{M}_{\mathcal{G}_{1} \rightarrow \mathcal{G}_{2}}$.

Similarly, for any $\mathcal{G}_{1}, \mathcal{G}_{2}$, if $\mathcal{G}_{1} \preceq_{\kappa_{1}}^{\text {cycle }} \mathcal{G}_{2}$ with the map $\mathcal{M}_{\mathcal{G}_{1} \rightarrow \mathcal{G}_{2}}$ and $\mathcal{G}_{2} \varsigma_{\kappa_{2}}^{\text {cycle }} \mathcal{G}_{3}$ with the map $\mathcal{M}_{\mathcal{G}_{2} \rightarrow \mathcal{G}_{3}}$, then $\mathcal{G}_{1} \preceq_{\kappa_{1} \kappa_{2}}^{\text {cycle }} \mathcal{G}_{3}$ with the map $\mathcal{M}_{\mathcal{G}_{1} \rightarrow \mathcal{G}_{3}}=\mathcal{M}_{\mathcal{G}_{2} \rightarrow \mathcal{G}_{3}} \circ \mathcal{M}_{\mathcal{G}_{1} \rightarrow \mathcal{G}_{2}}$.

The most important property of this is that this notion of approximation is also additive, i.e., it works well with graph decompositions.

Definition 3.11 (Union of two instances). Consider smoothed $p$ norm instances, $\mathcal{G}_{1}, \mathcal{G}_{2}$, with the same set of vertices, i.e. $V^{\mathcal{G}_{1}}=$ $V^{\mathcal{G}_{2}}$. Define $\mathcal{G}=\mathcal{G}_{1} \cup \mathcal{G}_{2}$ as the instance on the same set of vertices obtained by taking a disjoint union of the edges (potentially resulting in multi-edges). Formally,

$$
\mathcal{G}=\left(V^{\mathcal{G}_{1}}, E^{\mathcal{G}_{1}} \cup E^{\mathcal{G}_{2}},\left(\boldsymbol{g}^{\mathcal{G}_{1}}, \boldsymbol{g}^{\mathcal{G}_{2}}\right),\left(\boldsymbol{r}^{\mathcal{G}_{1}}, \boldsymbol{r}^{\mathcal{G}_{2}}\right),\left(s^{\mathcal{G}_{1}}, s^{\mathcal{G}_{2}}\right)\right) .
$$

LEMMA 3.12 ( $\leq_{\kappa}$ UNDER UNION). Consider four smoothed p-norm instances, $\mathcal{G}_{1}, \mathcal{G}_{2}, \mathcal{H}_{1}, \mathcal{H}_{2}$, on the same set of vertices, i.e. $V^{\mathcal{G}_{1}}=$ $V^{\mathcal{G}_{2}}=V^{\mathcal{H}_{1}}=V^{\mathcal{H}_{2}}$, such that for $\left.i=1,2, \mathcal{H}_{i}\right\rfloor_{\kappa} \mathcal{G}_{i}$ with the map 
$\mathcal{M}_{\mathcal{H}_{i} \rightarrow \mathcal{G}_{i}}$. Let $\mathcal{G} \stackrel{\text { def }}{=} \mathcal{G}_{1} \cup \mathcal{G}_{2}$, and $\mathcal{H} \stackrel{\text { def }}{=} \mathcal{H}_{1} \cup \mathcal{H}_{2}$. Then, $\mathcal{H} \unlhd_{\kappa} \mathcal{G}$ with the map

$\mathcal{M}_{\mathcal{H} \rightarrow \mathcal{G}}\left(f^{\mathcal{H}}=\left(f^{\mathcal{H}_{1}}, f^{\mathcal{H}_{2}}\right)\right) \stackrel{\text { def }}{=}\left(\mathcal{M}_{\mathcal{H}_{1} \rightarrow \mathcal{G}_{1}}\left(f^{\mathcal{H}_{1}}\right), \mathcal{M}_{\mathcal{H}_{2} \rightarrow \mathcal{G}_{2}}\left(f^{\mathcal{H}_{2}}\right)\right)$,

where $\left(f^{H_{1}}, f^{H_{2}}\right)$ is the decomposition of $f^{H}$ onto the supports of $H_{1}$ and $\mathrm{H}_{2}$.

This notion of approximation also behaves nicely with scaling of $\ell_{2}$ and $\ell_{p}$ resistances.

LEMMA 3.13. For all $\kappa \geq 1$, and for all pairs of smoothed p-norm instances, $\mathcal{G}, \mathcal{H}$, on the same underlying graphs, i.e., $\left(V^{\mathcal{G}},{ }^{\mathcal{G}}\right)=$ $\left(V^{\mathcal{H}}, E^{\mathcal{H}}\right)$, such that,

(1) the gradients are identical, $\mathbf{g}^{\mathcal{G}}=\mathbf{g}^{\mathcal{H}}$,

(2) the $\ell_{2}^{2}$ resistances are off by at most $\kappa$, i.e., $\boldsymbol{r}_{e}^{\mathcal{G}} \leq \kappa \boldsymbol{r}_{e}^{\mathcal{H}}$ for all edges $e$, and

(3) the p-norm scaling is off by at most $\kappa^{p-1}$, i.e., $s^{\mathcal{G}} \leq \kappa^{p-1} s^{\mathcal{H}}$, then $\mathcal{H} \leq_{\kappa} \mathcal{G}$ with the identity map.

\subsection{Orthogonal Decompositions of Flows}

At the core of our graph decomposition and sparsification procedures is a decomposition of the gradient $\boldsymbol{g}$ of $\mathcal{G}$ into its cycle space and potential flow space. We denote such a splitting using

$$
\mathbf{g}^{\mathcal{G}}=\widehat{\boldsymbol{g}}^{\mathcal{G}}+B^{\mathcal{G}} \psi^{\mathcal{G}} \text {, s.t. } B^{\mathcal{G}^{\top}} \widehat{\boldsymbol{g}}^{\mathcal{G}}=\mathbf{0} \text {. }
$$

Here $\widehat{\boldsymbol{g}}$ is a circulation, while $\boldsymbol{B} \boldsymbol{\psi}$ gives a potential induced edge value. We will omit the superscripts when the context is clear.

The following minimization based formulation of this splitting of $g$ is critical to our method of bounding the overall progress of our algorithm.

LEMMA 3.14. The projection of $\mathbf{g}$ onto the cycle space is obtained by minimizing the energy after adding a potential flow to $\mathrm{g}$. Specifically,

$$
\|\widehat{\mathbf{g}}\|_{2}^{2}=\min _{\boldsymbol{x}}\|\boldsymbol{g}+\boldsymbol{B} \boldsymbol{x}\|_{2}^{2}
$$

LEMma 3.15. Given a graph/gradient instance $\mathcal{G}$, consider $\mathcal{H}$ formed from a subset of its edges. The projections of $\mathrm{g}^{\mathcal{G}}$ and $\mathbf{g}^{\mathcal{H}}$ onto their respective cycle spaces, $\widehat{\mathbf{g}}^{\mathcal{G}}$ and $\widehat{\boldsymbol{g}}^{\mathcal{H}}$ satsify:

$$
\left\|\widehat{\boldsymbol{g}}^{\mathcal{H}}\right\|_{2}^{2} \leq\left\|\widehat{\boldsymbol{g}}^{\mathcal{G}}\right\|_{2}^{2} \leq\left\|\boldsymbol{g}^{\mathcal{G}}\right\|_{2}^{2}
$$

\subsection{Iterative Refinement}

The work of Adil et al. [2] proved that $p$-norm minimization problems could be iteratively refined. Following their approach, we can show that the class of smoothed $p$-norm minimization problems can be iteratively refined. The following key lemma allows us to approximate the change in the smoothed $p$-norm of $x+\delta$ relative to the norm of $x$, in terms of another smoothed $p$-norm of $\boldsymbol{\delta}$.

Lemma 3.16 ([2]). For all $\boldsymbol{r}, \boldsymbol{x}, \boldsymbol{\delta} \in \mathbb{R}^{m}$, with $\boldsymbol{r} \in \mathbb{R}_{\geq 0}^{m}$, and $s \geq 0$, we have

$$
\begin{aligned}
& 2^{-p} \cdot h_{p}\left(\boldsymbol{r}+|\boldsymbol{x}|^{p-2}, s, \boldsymbol{\delta}\right) \\
& \leq h_{p}(\boldsymbol{r}, s, \boldsymbol{x}+\boldsymbol{\delta})-h_{p}(\boldsymbol{r}, s, \boldsymbol{x})-\boldsymbol{\delta}^{\top} \nabla_{x} h_{p}(\boldsymbol{r}, s, \boldsymbol{x}) \\
& \leq 2^{2 p} \cdot h_{p}\left(\boldsymbol{r}+|\boldsymbol{x}|^{p-2}, s, \boldsymbol{\delta}\right) .
\end{aligned}
$$

The above lemma gives us the following theorem about iteratively refining smoothed $\ell_{p}$-norm minimization problems.

Theorem 3.17 ([2]). Given the following optimization problem,

$$
\begin{aligned}
\max _{\boldsymbol{x}} & \mathcal{E}_{1}(\boldsymbol{x}) \stackrel{\text { def }}{=} \boldsymbol{g}^{\top} \boldsymbol{x}-h_{p}(\boldsymbol{r}, s, \boldsymbol{x}) \\
\text { s.t. } & \boldsymbol{A x}=\boldsymbol{b}
\end{aligned}
$$

and an initial feasible solution $x_{0}$, we can construct the following residual problem:

$$
\begin{aligned}
\max _{\boldsymbol{\delta}} & \mathcal{E}_{2}(\boldsymbol{\delta}) \stackrel{\text { def }}{=}\left(\boldsymbol{g}^{\prime}\right)^{\top} \boldsymbol{\delta}-h_{p}\left(\boldsymbol{r}^{\prime}, s, \boldsymbol{\delta}\right) \\
\text { s.t. } & \boldsymbol{A} \boldsymbol{\delta}=\mathbf{0},
\end{aligned}
$$

where $\mathbf{g}^{\prime}=2^{p}\left(\boldsymbol{g}-\left.\nabla_{\boldsymbol{x}} h(\boldsymbol{r}, \boldsymbol{s}, \boldsymbol{x})\right|_{x=\boldsymbol{x}_{0}}\right)$, and $\boldsymbol{r}^{\prime}=\boldsymbol{r}+s\left|\boldsymbol{x}_{0}\right|^{p-2}$.

There exists a feasible solution $\widetilde{\delta}$ to the residual problem $R 1$ that achieves an objective of $\mathcal{E}_{2}(\widetilde{\boldsymbol{\delta}}) \geq 2^{p}\left(\mathcal{E}_{1}\left(x^{\star}\right)-\mathcal{E}_{1}\left(x_{0}\right)\right)$, where $x^{\star}$ is an optimal solution to problem $P 1$.

Moreover, given any feasible solution $\boldsymbol{\delta}$ to Program R1, the vector $x_{1} \stackrel{\text { def }}{=} x_{0}+2^{-3 p} \boldsymbol{\delta}$ is a feasible solution to the Program P1 and obtains the objective

$$
\mathcal{E}_{1}\left(x_{1}\right) \geq \mathcal{E}_{1}\left(x_{0}\right)+2^{-4 p} \mathcal{E}_{2}(\boldsymbol{\delta}) .
$$

Importantly, we can apply the above theorem to smoothed $p$ norm flow/circulation problems.

COROLLARY 3.18 (ITERATIVE REFINEMENT FOR SMOOTHED $p$-NORM FLOW/CIRCULATION PROBLEMS). Given any smoothed p-norm flow problem $(\mathcal{G}, \boldsymbol{b})$ with optimal objective $\mathcal{E}^{\star}(\mathcal{G})$, and any initial circulation $f_{0}$, we can build, in $O\left(\left|E^{\mathcal{G}}\right|\right)$ time, a smoothed p-norm circulation problem $\mathcal{H}$ with the same underlying graph $\left(V^{\mathcal{H}}, E^{\mathcal{H}}\right)=\left(V^{\mathcal{G}}, E^{\mathcal{G}}\right)$, such that $\mathcal{E}^{\star}(\mathcal{H}) \geq 2^{p}\left(\mathcal{E}^{\star}(\mathcal{G})-\mathcal{E}^{\mathcal{G}}\left(f_{0}\right)\right)$ and for any circulation $f^{\mathcal{H}}$ on $\mathcal{H}$, the flow $f_{1} \stackrel{\text { def }}{=} f_{0}+2^{-3 p} f^{\mathcal{H}}$ satisfies residues $\boldsymbol{b}$ on $\mathcal{G}$ and has an objective

$$
\mathcal{E}^{\mathcal{G}}\left(f_{1}\right) \geq \mathcal{E}^{\mathcal{G}}\left(f_{0}\right)+2^{-4 p} \mathcal{E}^{\mathcal{H}}\left(f^{\mathcal{H}}\right)
$$

\section{RECURSIVE PRECONDITIONING FOR FLOW PROBLEMS}

In this section, we present our main recursive preconditioning algorithm, RecursivePreconditioning (Algorithm 1). We discuss the subroutine UltraSparsify in Section 5, and the subroutines Eliminate, RemoveLoops, and SolveLoops in Section 6. Here is the key theorem about the performance of the algorithm.

Theorem 4.1 (Recursive Preconditioning). Given a smoothed $p$-norm instance $\mathcal{G}$ with $p \geq 2$, residues $\boldsymbol{b}$, initial solution $\boldsymbol{f}^{(0)}$, and $\delta \leq 1$ such that $\log 1 / \delta, \log \left\|\boldsymbol{g}^{\mathcal{G}}\right\|, \log \left\|\boldsymbol{r}^{\mathcal{G}}\right\|, \log \left\|f^{(0)}\right\| \leq \widetilde{O}(1)$. We can pick $\kappa=\widetilde{\Theta}\left(m^{\frac{1}{\sqrt{p-1}}}\right)$ so Algorithm 1 runs in time $2^{O\left(p^{3 / 2}\right)} m^{1+O\left(\frac{1}{\sqrt{p-1}}\right)}$, and returns a flow $f$ on $\mathcal{G}$ such that $f$ satisfies residues $\boldsymbol{b}$, and

$$
\mathcal{E}^{\star}(\mathcal{G})-\mathcal{E}^{\mathcal{G}}\left(f^{(T)}\right) \leq \frac{1}{2}\left(\mathcal{E}^{\star}(\mathcal{G})-\mathcal{E}^{\mathcal{G}}\left(f^{(0)}\right)\right)+\delta s^{\mathcal{G}} .
$$

We give a proof sketch of Theorem 4.1. For the sketch we ignore additive terms in our derivation, i.e. $\delta s^{\mathcal{G}}$, and we apply Theorem 5.1 ignoring the additive terms, i.e. $\delta\left\|f^{\mathcal{G}}\right\|_{2}\left\|g^{\mathcal{G}}\right\|_{2}$ and $\delta\left(\left\|f^{\mathcal{H}}\right\|_{2}\left\|g^{\mathcal{G}}\right\|_{2}+\right.$ $\left\|f^{\mathcal{H}}\right\|_{2}^{2}$ ). These terms are additive errors incurred by our analysis, and we can show that they are small throughout the algorithm. 


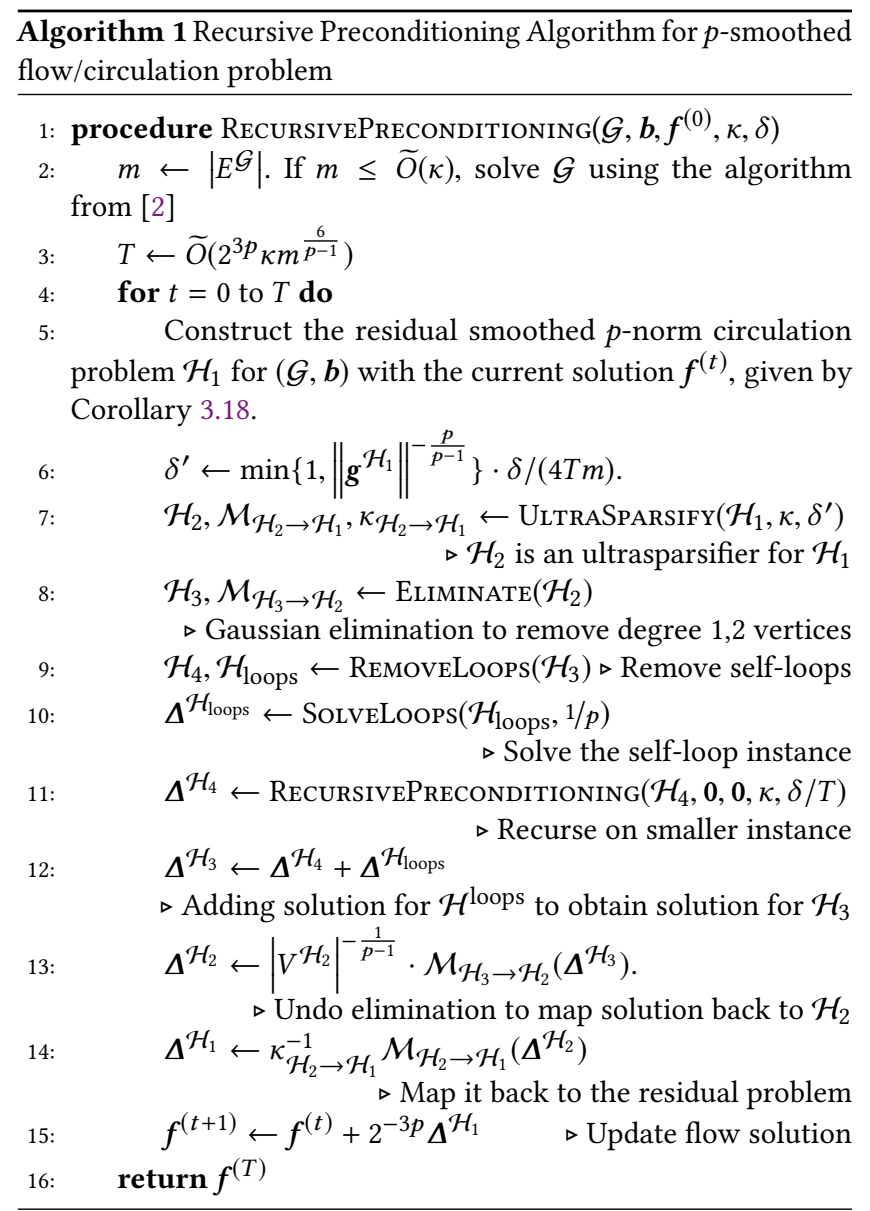

Although not fully accurate, the sketch captures the main ideas of our algorithm, while hiding the tedious details to deal with small additive errors. We also omit the details of the running time analysis in the sketch. We include the proof in the full version of our paper.

Proof SKetch of Theorem 4.1. By scaling $\boldsymbol{g}^{\mathcal{G}}, \boldsymbol{r}^{\mathcal{G}}$, we can assume that $s^{\mathcal{G}}=1$ without loss of generality.

Let us consider iteration $t$ of the for loop in Algorithm 1. First, let us prove guarantees on the optimal solutions of all the relevant instances. By the guarantees of Corollary 3.18, we know that $\mathcal{H}_{1}$ is a smoothed $p$-norm circulation problem with the same underlying graph $\left(V^{\mathcal{H}_{1}}, E^{\mathcal{H}_{1}}\right)=\left(V^{\mathcal{G}}, E^{\mathcal{G}}\right)$, such that $\mathcal{E}^{\star}\left(\mathcal{H}_{1}\right) \geq 2^{p}\left(\mathcal{E}^{\star}(\mathcal{G})-\right.$ $\left.\mathcal{E}^{\mathcal{G}}\left(f^{(t)}\right)\right)$.

From Theorem 5.1, we know that UltraSparsify returns a smoothed $p$-norm circulation instance $\mathcal{H}_{2}$ on the same set of vertices, and if we ignore the additive terms in the theorem statement, we have $\mathcal{E}^{\star}\left(\mathcal{H}_{2}\right) \geq \kappa_{\mathcal{H}_{1} \rightarrow \mathcal{H}_{2}}^{-1} \mathcal{E}^{\star}\left(\mathcal{H}_{1}\right)$

From Theorem 6.1, we know that the instance $\mathcal{H}_{3}$ returned by Eliminate $\left(\mathcal{H}_{2}\right)$ satisfies $\mathcal{H}_{2} \leq_{1}^{\text {cycle }} \mathcal{H}_{3}$, and hence $\mathcal{E}^{\star}\left(\mathcal{H}_{2}\right) \leq$ $\mathcal{E}^{\star}\left(\mathcal{H}_{3}\right)$. From Lemma 6.2 , we know that $\mathcal{E}^{\star}\left(\mathcal{H}_{4}\right)+\mathcal{E}^{\star}\left(\mathcal{H}_{\text {loops }}\right)=$ $\mathcal{E}^{\star}\left(\mathcal{H}_{3}\right)$. Combining these guarantees, we obtain,

$\mathcal{E}^{\star}\left(\mathcal{H}_{3}\right)=\mathcal{E}^{\star}\left(\mathcal{H}_{4}\right)+\mathcal{E}^{\star}\left(\mathcal{H}_{\text {loops }}\right) \geq 2^{p} \kappa_{\mathcal{H}_{1} \rightarrow \mathcal{H}_{2}}^{-1}\left(\mathcal{E}^{\star}(\mathcal{G})-\mathcal{E}^{\mathcal{G}}\left(f^{(t)}\right)\right)$
Now, we analyze the approximation guarantee provided by the solutions to these instances. From Lemma 6.2 , SolveLoops $\left(\mathcal{H}_{\text {loops }}\right)$ returns a $\Delta^{\mathcal{H}_{\text {loops }}}$ that satisfies $\mathcal{E}^{\mathcal{H}_{\text {loops }}}\left(\Delta^{\mathcal{H}_{\text {loops }}}\right) \geq \frac{1}{2} \mathcal{E}^{\star}\left(\mathcal{H}_{\text {loops }}\right)$. By induction, RecursivePreconditioning $\left(\mathcal{H}_{4}, \mathbf{0}, \kappa, \delta T^{-1}\right)$, upon starting with the initial solution 0 , returns a $\Delta^{\mathcal{H}_{4}}$ that satisfies, $\mathcal{E}^{\mathcal{H}_{4}}\left(\Delta^{\mathcal{H}_{4}}\right) \geq \frac{1}{2} \mathcal{E}^{\star}\left(\mathcal{H}_{4}\right)$, where we again ignore the term $-\delta T^{-1}{ }_{s} \mathcal{G}$ in this sketch. Combining these guarantees, we have,

$$
\mathcal{E}^{\mathcal{H}_{3}}\left(\Delta^{\mathcal{H}_{3}}\right)=\mathcal{E}^{\mathcal{H}_{4}}\left(\Delta^{\mathcal{H}_{4}}\right)+\mathcal{E}^{\mathcal{H}_{\text {loops }}}\left(\Delta^{\mathcal{H}_{\text {loops }}}\right) \geq \frac{1}{2} \mathcal{E}^{\star}\left(\mathcal{H}_{3}\right)
$$

From Theorem 6.1, we also have $\mathcal{H}_{3} \leq_{\kappa_{\text {elim }}}^{\text {cycle }} \mathcal{H}_{2}$, for $\kappa_{\text {elim }}=\left|V^{\mathcal{H}_{2}}\right|^{\frac{1}{p-1}}$, and hence

$$
\kappa_{\text {elim }}^{-1} \mathcal{E}^{\mathcal{H}_{3}}\left(\Delta^{\mathcal{H}_{3}}\right) \leq \mathcal{E}^{\mathcal{H}_{2}}\left(\kappa_{\mathrm{elim}}^{-1} \mathcal{M}_{\mathcal{H}_{3} \rightarrow \mathcal{H}_{2}}\left(\Delta^{\mathcal{H}_{3}}\right)\right)=\mathcal{E}^{\mathcal{H}_{2}}\left(\Delta^{\mathcal{H}_{2}}\right) .
$$

Finally, from Theorem 5.1 (but with additive errors ignored), we have,

$$
\begin{aligned}
\mathcal{E}^{\mathcal{H}_{1}}\left(\Delta^{\mathcal{H}_{1}}\right) & =\mathcal{E}^{\mathcal{H}_{1}}\left(\kappa_{\mathcal{H}_{2} \rightarrow \mathcal{H}_{1}}^{-1} \mathcal{M}_{\mathcal{H}_{2} \rightarrow \mathcal{H}_{1}}\left(\Delta^{\mathcal{H}_{2}}\right)\right) \\
& \geq \kappa_{\mathcal{H}_{2} \rightarrow \mathcal{H}_{1}}^{-1} \mathcal{E}^{\mathcal{H}_{2}}\left(\Delta^{\mathcal{H}_{2}}\right)
\end{aligned}
$$

Combining these guarantees, we obtain,

$$
\begin{aligned}
\mathcal{E}^{\mathcal{H}_{1}}\left(\Delta^{\mathcal{H}_{1}}\right) & \geq \kappa_{\mathcal{H}_{2} \rightarrow \mathcal{H}_{1}}^{-1} \kappa_{\operatorname{elim}}^{-1}\left(\frac{1}{2} 2^{p} \kappa_{\mathcal{H}_{1} \rightarrow \mathcal{H}_{2}}^{-1}\left(\mathcal{E}^{\star}(\mathcal{G})-\mathcal{E}^{\mathcal{G}}\left(f^{(t)}\right)\right)\right) \\
& \geq \widetilde{\Omega}\left(2^{p} \kappa^{-1} m^{-\frac{6}{p-1}}\right)\left(\mathcal{E}^{\star}(\mathcal{G})-\mathcal{E}^{\mathcal{G}}\left(f^{(t)}\right)\right) .
\end{aligned}
$$

Thus, by Corollary 3.18, $f^{(t+1)}$ satisfies

$$
\begin{aligned}
& \mathcal{E}^{\star}(\mathcal{G})-\mathcal{E}^{\mathcal{G}}\left(f^{(t+1)}\right) \\
\leq & \mathcal{E}^{\star}(\mathcal{G})-\mathcal{E}^{\mathcal{G}}\left(f^{(t)}\right)-2^{-4 p} \mathcal{E}^{\mathcal{H}_{1}}\left(\Delta^{\mathcal{H}_{1}}\right) \\
\leq & \left(1-\widetilde{\Omega}\left(2^{-3 p} \kappa^{-1} m^{-\frac{6}{p-1}}\right)\right)\left(\mathcal{E}^{\star}(\mathcal{G})-\mathcal{E}^{\mathcal{G}}\left(f^{(t)}\right)\right) .
\end{aligned}
$$

Thus, repeating for loop $\widetilde{O}\left(2^{3 p_{\kappa}} \frac{6}{p-1}^{\frac{6}{p}}\right)$ times gives us a solution $f^{(T)}$ such that

$$
\mathcal{E}^{\star}(\mathcal{G})-\mathcal{E}^{\mathcal{G}}\left(f^{(T)}\right) \leq \frac{1}{2}\left(\mathcal{E}^{\star}(\mathcal{G})-\mathcal{E}^{\mathcal{G}}\left(f^{(0)}\right)\right) .
$$

Now, we analyze the running time. In a single iteration, the total cost of all the operations other than the recursive call to RecursivePreconditioning is $\widetilde{O}(m)$. Using the guarantees on size of the instances $\mathcal{H}_{1}, \mathcal{H}_{2}$, and $\mathcal{H}_{4}$, we get the total running time recurrence is

$$
T(m) \leq \widetilde{O}\left(2^{3 p} \kappa m^{\frac{6}{p-1}}\right)(T(m / \kappa)+\widetilde{O}(m)) .
$$

Note that $\kappa$ is fixed throughout the recursion. By picking $\kappa=$ $\widetilde{\Theta}\left(m^{\frac{1}{\sqrt{p-1}}}\right)$, we can fix the depth of the recursion to be $O(\sqrt{p-1})$. The total cost is dominated by the cost at the bottom level of the recursion, which adds up to a total running time of $2 O\left(p^{3 / 2}\right) m^{1+O\left(\frac{1}{\sqrt{p-1}}\right)}$.

\section{ULTRA-SPARSIFICATION}

In this section, we discuss at a high level of the construction of ultra-sparsifiers for a smooth $p$-norm instance. We defer the full analysis to the full version of the paper. We start with the main theorem of our ultra-sparsifier. 
Theorem 5.1. Given any instance $\mathcal{G}=\left(V^{\mathcal{G}}, E^{\mathcal{G}}, \boldsymbol{g}^{\mathcal{G}}, \boldsymbol{r}^{\mathcal{G}},{ }_{s}^{\mathcal{G}}\right)$ with $n$ nodes, $m$ edges, and parameters $\kappa, \delta$ where $\log \frac{1}{\delta}$ and $\log \left\|\boldsymbol{r}^{\mathcal{G}}\right\|_{\infty}$ are both $O\left(\log ^{c} n\right)$ for some constant $c$ and $\kappa<m$, ULTRASPARSIFY computes in $\widetilde{O}(m)$ running time another instance $\mathcal{H}=\left(V^{\mathcal{H}}, E^{\mathcal{H}}, \boldsymbol{g}^{\mathcal{H}}\right.$, $\boldsymbol{r}^{\mathcal{H}}, s^{\mathcal{H}}=s^{\mathcal{G}}$ ) along with flow mapping functions $\mathcal{M}_{\mathcal{H} \rightarrow \mathcal{G}}, \mathcal{M}_{\mathcal{G} \rightarrow \mathcal{H}}$ such that $V^{\mathcal{H}}=V^{\mathcal{G}}$, and with high probability we have

(1) $E^{H}$ consists of a spanning tree in the graph $\left(V^{\mathcal{G}}, E^{\mathcal{G}}\right)$, up to $m-n+1$ self-loops and at most $\widetilde{O}\left(\frac{m}{\kappa}\right)$ other non self-loop edges.

(2) With $\kappa_{\mathcal{G} \rightarrow \mathcal{H}}=\widetilde{O}\left(\kappa^{3 /(p-1)}\right)$ for any flow $f^{\mathcal{G}}$ of $\mathcal{G}$ we have

$\mathcal{E}_{\mathcal{H}}\left(\frac{\mathcal{M}_{\mathcal{G} \rightarrow \mathcal{H}}\left(f^{\mathcal{G}}\right)}{\kappa_{\mathcal{G} \rightarrow \mathcal{H}}}\right) \geq \frac{1}{\kappa_{\mathcal{G} \rightarrow \mathcal{H}}} \mathcal{E}_{\mathcal{G}}\left(f^{\mathcal{G}}\right)-\delta\left\|f^{\mathcal{G}}\right\|_{2}\left\|\boldsymbol{g}^{\mathcal{G}}\right\|_{2}$,

and with $\kappa_{\mathcal{H} \rightarrow \mathcal{G}}=\widetilde{O}\left(m^{2 /(p-1)}\right)$, for any flow solution $f^{\mathcal{H}}$ of $\mathcal{H}$ we have

$\mathcal{E}_{\mathcal{G}}\left(\frac{\mathcal{M}_{\mathcal{H} \rightarrow \mathcal{G}}\left(f^{\mathcal{H}}\right)}{\kappa_{\mathcal{H} \rightarrow \mathcal{G}}}\right) \geq \frac{1}{\kappa_{\mathcal{H} \rightarrow \mathcal{G}}} \mathcal{E}_{\mathcal{H}}\left(f^{\mathcal{H}}\right)-\delta\left(\left\|f^{\mathcal{H}}\right\|_{2}\left\|g^{\mathcal{G}}\right\|_{2}+\left\|f^{\mathcal{H}}\right\|_{2}^{2}\right)$.

The flow mappings $\mathcal{M}_{\mathcal{H} \rightarrow \mathcal{G}}, \mathcal{M}_{\mathcal{G} \rightarrow \mathcal{H}}$ preserve residue of flow, and can be applied in $\widetilde{O}(m)$ time.

\subsection{Overview}

Our high-level approach is the same as Spielman-Teng [67], where we utilize a low-stretch spanning tree, and move off-tree edges to a small set of portal nodes. Once most off-tree edges are only between a small set of portal nodes, we sparsify the graph over the portal nodes to reduce the number of edges. As we need to map flow solutions between the original instance and the sparsified instance, our main concern is to carry out these step without incurring too much error on the objective function values. In our case we have $\ell_{p}^{p}$ resistances and gradients on edges in addition to $\ell_{2}^{2}$ resistances, and thus the main challenge is to simultaneously preserve their respective terms.

Tree-portal Routing. We first formalize what we mean by moving off-tree edges. Suppose we have a spanning tree $T$ of a graph $(V, E)$ and a subset set of nodes $\widehat{V} \subset V$ designated as portal nodes, for any off-tree edge $e=\{u, v\} \in E \backslash T$, there is a unique tree path $\mathcal{P}_{T}(u, v)$ in $T$ from $u$ to $v$. We define a tree-portal path $\mathcal{P}_{T, \widehat{V}}(u, v)$, which is not necessarily a simple path.

Definition 5.2 (Tree-portal path and edge moving). Given spanning tree $T$ and set of portal nodes $\widehat{V}$, let $e=\{u, v\}$ be any edge not in $T$, and $P_{T}(u, v)$ the unique tree path in $T$ from $u$ to $v$. We define $e$ 's tree-portal path $P_{T, \widehat{V}}(u, v)$ and $e$ 's image under tree-portal edge moving as follows

(1) If $P_{T}(u, v)$ doesn't go through any portal vertex. In this case, we replace $\{u, v\}$ with a distinct self-loop of $v$. We let $P_{T, \widehat{V}}(u, v)$ be the path $P_{T}(u, v)$ followed by the self-loop at $v$.

(2) If $P_{T}(u, v)$ goes through exactly one portal vertex $\widehat{v}$. In this case, we replace $\{u, v\}$ with a distinct self-loop at $\widehat{v}$. We let $P_{T, \widehat{V}}(u, v)$ be the tree path $P_{T}(u, \widehat{v})$ followed by the self-loop at $\widehat{v}$ and then the tree path $P_{T}(\widehat{v}, v)$.
(3) If $P_{u v}$ goes through at least two portal vertices. In this case, let $\widehat{u}$ (and $\widehat{v}$ ) be closest the portal vertex to $u$ (and $v$ ) on $P_{u v}$, we replace $\{u, v\}$ with a distinct $\operatorname{edge}^{2}\{\widehat{u}, \widehat{v}\}$. We let $P_{T, \widehat{V}}(u, v)$ be the tree path $P_{T}(u, \widehat{u})$ followed by the new edge from $\widehat{u}$ to $\widehat{v}$ and then the tree path $P_{T}(\widehat{v}, v)$.

This maps any off-tree edge $e$ to a unique (edge or self-loop) $\widehat{e}$ given any $T, \widehat{V}$. We denote the tree-portal edge moving with the map $\widehat{e}=\operatorname{MovE}_{T, \widehat{V}}(e)$.

Although we will get self-loops in tree-portal routing, to keep the discussion simple, we ignore the possibility of getting self-loops. This still captures all the main ideas, and the algorithm/analysis extends to self-loops in a very straightforward but slightly tedious way. We discuss self-loops briefly at the end of the section.

Tree-portal routing is a mapping from flow solutions on the original off-tree edges to a flow solution (with the same residue) using the edges they are mapped to. Any flow along off-tree edge $(u, v)$ in the original graph is rerouted (again from $u$ to $v$ ) using the tree-portal path $P_{T, \widehat{V}}(u, v)$ instead. Rerouting the flow of any off-tree edge along its tree-portal path increases the congestion on tree edges, which in turn incurs error in the $\ell_{2}^{2}$ and $\ell_{p}^{p}$ terms in the objective function. We need to pick the tree and portal nodes carefully to bound the error.

Definition 5.3. Given any graph $(V, E)$, resistance $r$ on edges, a spanning tree $T$, and a set of portals $\widehat{V} \subset V$, for any $e=\{u, v\} \in E$, let $\widehat{e}=\operatorname{MovE}_{T, \widehat{V}}(e)$ and $P_{T, \widehat{V}}(u, v)$ be as specified above. The stretch of $e=\{u, v\} \in E \backslash T$ in the tree-portal routing is

$$
\operatorname{Str}_{T, \widehat{V}}(e) \stackrel{\text { def }}{=} \frac{1}{\boldsymbol{r}_{e}} \sum_{e^{\prime} \in P_{T, \widehat{V}}(e) \backslash\{\widehat{e}\}} \boldsymbol{r}_{e^{\prime}},
$$

and the stretch of a tree edge $e \in T$ is $\operatorname{Str}_{T, \widehat{V}}(e)=1$. Note with our definition $\operatorname{Str}_{T, \emptyset}(e)$ gives the standard stretch.

The starting point is low stretch spanning trees [1], which provide good bounds on the total $\ell_{2}^{2}$ stretch.

Lemma 5.4 (Low-Stretch Trees [1]). Given any graph $G=$ $(V, E)$ of $m$ edges and $n$ nodes, as well as resistance $\boldsymbol{r}, \operatorname{LSST}(\boldsymbol{r})$ finds a spanning tree in $O(m \log n \log \log n)$ time such that

$$
\sum_{e \in E} \operatorname{Str}_{T, \emptyset}(e) \leq O(m \log n \log \log n) .
$$

We will construct a low-stretch spanning tree $T$ of $\left(V^{\mathcal{G}}, E^{\mathcal{G}}, \boldsymbol{r}^{\mathcal{G}}\right)$ using the above result. Still, the error will be too large if we only use tree edges to reroute the flow of all the off-tree edges, since the low average stretch doesn't prevent one tree edge to be on the tree path for many off-tree edges. Thus, we need to add portal nodes so we can shortcut between them to reduce the extra congestion on tree edges. We can find a small set of good portal nodes so that rerouting flow on off-tree edges using their tree-portal paths incurs small error in the objective function.

\footnotetext{
${ }^{2}$ We will keep multi-edges explicitly between portal nodes.
} 
Graph Decomposition. Tree-portal routing will give us an instance where all the off-tree edges are between portal nodes, and we can look at the sub-graph restricted to the portal nodes and the off-tree edges between them. This graph has many fewer nodes comparing to the original graph but roughly the same number of edges, and thus is much denser. We can then sparsify this graph to reduce the number of off-tree edges similar to the construction of spectral sparsifiers. The main technical difficulty is that in the sparsified graph, we still want the $\ell_{p}^{p}$ terms in our objective function to have a same scalar $s$ for every edge, but similar to the case of how resistances are scaled in spectral sparsification, to preserve the total value of the $\ell_{p}^{p}$ terms, we would naturally want to scale $s$ according to the probability we sample an edge $e$. Thus, to get a same scalar $s$ for all sampled edges, we are limited to uniform sampling. We know uniform sampling works in expanders, so the natural approach is to first decompose the graph into expanders, and sampling uniformly inside each expander as in $[35,66]$. However, because of the presence of a gradient, we need to be a bit more careful than even expander-decomposition based sparsification steps. Thus, we work with uniform expanders.

Definition 5.5. A graph ${ }^{3} G$ is a $\alpha$-uniform $\phi$-expander (or uniform expander when parameters not spelled out explicitly) if

(1) $r$ on all edges are the same.

(2) $s$ on all edges are the same.

(3) $G$ has conductance $e^{4}$ at least $\phi$.

(4) The projection of $\boldsymbol{g}$ onto the cycle space of $G, \widehat{\boldsymbol{g}}^{G}=(I-$ $\left.\boldsymbol{B} \boldsymbol{L}^{\dagger} \boldsymbol{B}^{\top}\right) \boldsymbol{g}$, is $\alpha$-uniform (see next definition), where $\boldsymbol{B}$ is the edge-vertex incidence matrix of $G$, and $L=B^{\top} B$ is the Laplacian.

Definition 5.6. A vector $y \in \mathbb{R}^{m}$ is said to be $\alpha$-uniform if

$$
\|y\|_{\infty}^{2} \leq \frac{\alpha}{m}\|y\|_{2}^{2}
$$

We abuse the notation to also let the all zero vector 0 be 1 -uniform.

Decomposing into Uniform Expanders. We can decompose the graph consisting of portals and the off-tree edges between them into vertex disjoint uniform expanders such that more than half of the edges are inside the expanders. Moreover, the min degree of any node in the expanders is up to a polylog factor close to the average degree. For the off-tree edges not included in these uniform expanders, we work on the pre-image ${ }^{5}$ of them in the next iteration. That is, for any edge $\widehat{e}$ inside one of the expanders, we remove its pre-image from the instance $\mathcal{G}$, and work on the remaining off-tree edges in $\mathcal{G}$ in the next iteration. This iterative process terminates when the number of remaining off-tree edges is small enough (i.e. $\left.\widetilde{O}\left(\left|E^{\mathcal{G}}\right| / \kappa\right)\right)$. This takes $O\left(\log \left|E^{\mathcal{G}}\right|\right)$ iterations as a constant fraction of off-tree edges are moved to be inside the expanders each iteration.

\footnotetext{
${ }^{3}$ We use an instance and its underlying graph interchangeably in our discussion. ${ }^{4} \boldsymbol{r}$ are uniform, so conductance is defined as in unweighted graphs. We use the standard definition of conductance. For graph $G=(V, E)$, the conductance of any $\emptyset \neq S \subsetneq V$ is $\phi(S)=\frac{\delta(S)}{\min (\operatorname{vol}(S), v o l(V \backslash S))}$ where $\delta(S)$ is the number of edges on the cut $(S, V \backslash S)$ and $\operatorname{vol}(S)$ is the sum of the degree of nodes in $S$. The conductance of a graph is $\phi_{G}=\min _{S \neq \emptyset, V} \phi(S)$.

${ }^{5} \mathrm{By}$ pre-image of $\widehat{e}$ we mean the original off-tree edge $e$ that gets moved to $\widehat{e}$ in the tree-portal routing, i.e. $e=\operatorname{MovE}_{T, \widehat{V}}^{-1}(\widehat{e})$.
}

Sparsify Uniform Expanders. If we append a column containing the gradient of edges to the edge-vertex incidence matrix $B$, the conditions of a $\alpha$-uniform $\phi$-expander is equivalent to each row of $\boldsymbol{B}$ having leverage score at most $\frac{n \alpha \phi^{-1}}{m}$ where $n, m$ are number of nodes and edges. An underlying connection with the $p$-norm row sampling result by Cohen and Peng [18] is that this is also a setting under which $q$-norm functionals are preserved under uniform sampling. We refrain from developing a more complete picture of such machinery here, and will utilize ideas closer to routing on expanders $[34,35]$ to show a cruder approximation.

We defer further details of the construction of our ultra-sparsifiers to the full version.

\section{ELIMINATION OF LOW-DEGREE VERTICES, AND LOOPS}

In this section, we take the instance $\mathcal{H}$ returned by UltraSparsify and repeatedly eliminate the vertices of degree at most 2 , to obtain a smaller graph. This step is analogous to the partial Cholesky factorization in the Laplacian solver of Spielman and Teng [67]. A slight technical issue is that if we run into a cycle where at most 1 vertex on the cycle has edge(s) to the rest of the graph, the elimination of the degree 2 nodes on the cycle essentially becomes an optimization problem on only the cycle edges that can be solved independently from the rest of the graph. We state the main results, and leave the details to the full version.

Theorem 6.1 (Eliminating vertices with Degree 1 And 2). Given a smoothed p-norm instance $\mathcal{G}$, the algorithm ELIMINATE $(\mathcal{G})$ returns another smoothed $p$-norm instance $\mathcal{G}^{\prime}$, along with the map $\mathcal{M}_{\mathcal{G}^{\prime} \rightarrow \mathcal{G}}$ in $O\left(\left|V^{\mathcal{G}}\right|+\left|E^{\mathcal{G}}\right|\right)$ time, such that the graph $G^{\prime}=\left(V^{\mathcal{G}^{\prime}}, E^{\mathcal{G}^{\prime}}\right)$ is obtained from the graph $G=\left(V^{\mathcal{G}}, E^{\mathcal{G}}\right)$ by first repeatedly removing vertices with non-selfloop degree 1 in $G$, and then replacing every path $u \leadsto v$ in $G$ where all internal path vertices have non-selfloop degree exactly 2 in $G$, with a new edge $(u, v)$.

Moreover,

$$
\mathcal{G}^{\prime} \leq_{n \frac{1}{p^{-1}}}^{\text {cycle }} \mathcal{G} \leq_{1}^{\text {cycle }} \mathcal{G}^{\prime},
$$

where $n=\left|V^{\mathcal{G}}\right|$, and $\mathcal{M}_{\mathcal{G}^{\prime} \rightarrow \mathcal{G}}$ can be applied in $O\left(\left|V^{\mathcal{G}}\right|+\left|E^{\mathcal{G}}\right|\right)$ time.

Lemma 6.2 (Eliminating Self-Loops). Given a smoothed p-norm instance $\mathcal{G}$ with self-loops in $E^{\mathcal{G}}$, the algorithm REMOVELOOPS, in $O\left(\left|V^{\mathcal{G}}\right|+\left|E^{\mathcal{G}}\right|\right)$ time, returns instances $\mathcal{G}_{1}, \mathcal{G}_{2}$, such that $\mathcal{G}=\mathcal{G}_{1} \cup \mathcal{G}_{2}$, where $\mathcal{G}_{1}$ is obtained from $\mathcal{G}$ by eliminating all self-loops from $E^{\mathcal{G}}$, and $\mathcal{G}_{2}$ is an instance consisting of just the self-loops from $\mathcal{G}$. Thus, any flow $f^{\mathcal{G}_{2}}$ on $\mathcal{G}_{2}$ is a circulation.

Moreover, there is an algorithm SOLVELoops that, given $\mathcal{G}_{2}$, for any $\delta \leq 1 / p$, in time $O\left(\left|E^{\mathcal{G}_{2}}\right| \log 1 / \delta\right)$, finds a circulation $\widetilde{f}^{\mathcal{G}_{2}}$ on $\mathcal{G}_{2}$ such that

$$
\mathcal{E}^{\mathcal{G}_{2}}\left(\tilde{f}^{\mathcal{G}_{2}}\right) \geq(1-\delta) \max _{f^{\mathcal{G}}:(\boldsymbol{B})^{\mathcal{G}} \boldsymbol{f}^{\mathcal{G}}=\mathbf{0}} \mathcal{E}^{\mathcal{G}_{2}}\left(f^{\mathcal{G}_{2}}\right)
$$

\section{REFERENCES}

[1] Ittai Abraham and Ofer Neiman. 2012. Using Petal-decompositions to Build a Low Stretch Spanning Tree. In STOC.

${ }^{6}$ By non-selfloop degree, we mean that self-loops do not count towards the degree of a vertex. 
[2] Deeksha Adil, Rasmus Kyng, Richard Peng, and Sushant Sachdeva. 2019. Iterative Refinement for $\ell_{p}$-norm Regression. In Proceedings of ACM-SIAM SODA 2019.

[3] Ravindra K. Ahuja, Thomas L. Magnanti, and James B. Orlin. 1993. Network flows - theory, algorithms and applications. Prentice Hall.

[4] Morteza Alamgir and Ulrike V Luxburg. 2011. Phase transition in the family of p-resistances. In Advances in Neural Information Processing Systems. 379-387.

[5] Zeyuan Allen-Zhu, Yuanzhi Li, Rafael Mendes de Oliveira, and Avi Wigderson. 2017. Much Faster Algorithms for Matrix Scaling. In Symposium on Foundations of Computer Science (FOCS). 890-901.

[6] Zeyuan Allen Zhu, Zhenyu Liao, and Lorenzo Orecchia. 2015. Spectral Sparsification and Regret Minimization Beyond Matrix Multiplicative Updates. In Proceedings of ACM STOC 2015. 237-245.

[7] N. Alon, R. M. Karp, D. Peleg, and D. West. 1995. A Graph-Theoretic Game and Its Application to the $k$-Server Problem. SIAM 7. Comput. 24, 1 (Feb. 1995), 78-100.

[8] David G. Anderson, Ming Gu, and Christopher Melgaard. 2014. An Efficient Algorithm for Unweighted Spectral Graph Sparsification. CoRR abs/1410.4273 (2014).

[9] Maxim A. Babenko, Jonathan Derryberry, Andrew V. Goldberg, Robert Endre Tarjan, and Yunhong Zhou. 2007. Experimental Evaluation of Parametric MaxFlow Algorithms. In WEA 2007,. 256-269.

[10] András A. Benczúr and David R. Karger. 1996. Approximating s-t minimum cuts in $\tilde{O}\left(n^{2}\right)$ time. In ACM STOC 1996. 47-55.

[11] Sébastien Bubeck, Michael B. Cohen, Yin Tat Lee, and Yuanzhi Li. 2018. An Homotopy Method for Lp Regression Provably Beyond Self-concordance and in Input-sparsity Time. In Proceedings of ACM STOC 2018 (STOC 2018). 1130-1137.

[12] Hui Han Chin, Aleksander Madry, Gary L. Miller, and Richard Peng. 2013. Runtime guarantees for regression problems. In ITCS 2013. 269-282.

[13] Paul Christiano, Jonathan A. Kelner, Aleksander Madry, Daniel A. Spielman, and Shang-Hua Teng. 2011. Electrical flows, laplacian systems, and faster approximation of maximum flow in undirected graphs. In ACM STOC 2011. 273-282.

[14] T. Chu, Y. Gao, R. Peng, S. Sachdeva, S. Sawlani, and J. Wang. 2018. Graph Sparsification, Spectral Sketches, and Faster Resistance Computation, via Short Cycle Decompositions. In IEEE FOCS 2018. 361-372.

[15] M. B Cohen, J. Kelner, R. Kyng, J. Peebles, R. Peng, A. B Rao, and A. Sidford. 2018 Solving Directed Laplacian Systems in Nearly-Linear Time through Sparse LU Factorizations. In IEEE FOCS 2018.

[16] M. B Cohen, J. Kelner, J. Peebles, R. Peng, A. B Rao, A. Sidford, and A. Vladu. 2017 Almost-linear-time algorithms for Markov chains and new spectral primitives for directed graphs. In ACM STOC 2017.

[17] M. B. Cohen, A. Madry, D. Tsipras, and A. Vladu. 2017. Matrix Scaling and Balancing via Box Constrained Newton's Method and Interior Point Methods. In IEEE FOCS 2017. 902-913.

[18] Michael B. Cohen and Richard Peng. 2015. $\ell_{p}$ Row Sampling by Lewis Weights. In Proceedings of ACM STOC. 183-192.

[19] S. I. Daitch and D. A. Spielman. 2008. Faster approximate lossy generalized flow via interior point algorithms. In ACM STOC 2008.

[20] Jack Edmonds. 1965. Paths, trees, and flowers. Canadian fournal of mathematics 17, 3 (1965), 449-467. Available at: https://cms.math.ca/10.4153/CJM-1965-045-4.

[21] Jack Edmonds and Richard M. Karp. 1972. Theoretical Improvements in Algorithmic Efficiency for Network Flow Problems. 7. ACM 19, 2 (1972), 248-264.

[22] Ahmed El Alaoui, Xiang Cheng, Aaditya Ramdas, Martin J Wainwright, and Michael I Jordan. 2016. Asymptotic behavior of $\ell_{p}$-based Laplacian regularization in semi-supervised learning. In Conference on Learning Theory. 879-906.

[23] Shimon Even and Robert Endre Tarjan. 1975. Network Flow and Testing Graph Connectivity. SIAM f. Comput. 4, 4 (1975), 507-518.

[24] Zvi Galil and Amnon Naamad. 1979. Network Flow and Generalized Path Compression. In ACM STOC 1979. 13-26.

[25] Giorgio Gallo, Michael D. Grigoriadis, and Robert Endre Tarjan. 1989. A Fast Parametric Maximum Flow Algorithm and Applications. SIAM f. Comput. 18, 1 (1989), 30-55

[26] Andrew V. Goldberg and Satish Rao. 1998. Beyond the Flow Decomposition Barrier. F. ACM 45, 5 (1998), 783-797.

[27] Andrew V. Goldberg and Robert Endre Tarjan. 1988. A new approach to the maximum-flow problem. 7. ACM 35, 4 (1988), 921-940.

[28] Andrew V. Goldberg and Robert Endre Tarjan. 2014. Efficient maximum flow algorithms. Commun. ACM 57, 8 (2014), 82-89.

[29] Dorit S Hochbaum. 2008. The pseudoflow algorithm: A new algorithm for the maximum-flow problem. Operations research 56, 4 (2008), 992-1009.

[30] Dorit S. Hochbaum and James B. Orlin. 2013. Simplifications and speedups of the pseudoflow algorithm. Networks 61, 1 (2013), 40-57.

[31] John E. Hopcroft and Richard M. Karp. 1973. An $n^{5 / 2}$ Algorithm for Maximum Matchings in Bipartite Graphs. SIAM F. Comput. 2, 4 (1973), 225-231.

[32] David R. Karger and Clifford Stein. 1996. A New Approach to the Minimum Cut Problem. 7. ACM 43, 4 (1996), 601-640.

[33] Alexander V. Karzanov. 1973. O nakhozhdenii maksimalńogo potoka v setyakh spetsialńogo vida i nekotorykh prilozheniyakh. Matematicheskie Voprosy Upravleniya Proizvodstvom 5 (1973), 81-94. In Russian, title translation: on finding maximum flows in networks with special structure and some applications.

[34] J.A. Kelner and A. Madry. 2009. Faster generation of random spanning trees. In FOCS.

[35] Jonathan A. Kelner, Yin Tat Lee, Lorenzo Orecchia, and Aaron Sidford. 2014. An Almost-Linear-Time Algorithm for Approximate Max Flow in Undirected Graphs, and its Multicommodity Generalizations. In ACM-SIAM SODA 2014. 217-226.

[36] J. A. Kelner, L. Orecchia, A. Sidford, and Z. A. Zhu. 2013. A simple, combinatoria algorithm for solving sdd systems in nearly-linear time. In STOC.

[37] Vladimir Kolmogorov, Yuri Boykov, and Carsten Rother. 2007. Applications of parametric maxflow in computer vision. In Computer Vision, 2007. ICCV 2007. IEEE 11th International Conference on. IEEE, 1-8.

[38] Ioannis Koutis, Gary L. Miller, and Richard Peng. 2011. A Nearly-m log n Time Solver for SDD Linear Systems. In IEEE FOCS 2011. 590-598.

[39] Ioannis Koutis, Gary L. Miller, and Richard Peng. 2012. A Fast Solver for a Class of Linear Systems. Commun. ACM 55, 10 (Oct. 2012), 99-107.

[40] R. Kyng, Y. T. Lee, R. Peng, S. Sachdeva, and D. A Spielman. 2016. Sparsified Cholesky and multigrid solvers for connection laplacians. In ACM STOC 2016.

[41] R. Kyng, A. B. Rao, S. Sachdeva, and D. A Spielman. 2015. Algorithms for Lipschitz learning on graphs. In COLT.

[42] R. Kyng and S. Sachdeva. 2016. Approximate Gaussian Elimination for Laplacians - Fast, Sparse, and Simple. In IEEE FOCS 2016. 573-582.

[43] Y. T. Lee, R. Peng, and D. A. Spielman. 2015. Sparsified Cholesky Solvers for SDD linear systems. CoRR abs/1506.08204 (2015)

[44] Y. T. Lee and A. Sidford. 2013. Efficient Accelerated Coordinate Descent Methods and Faster Algorithms for Solving Linear Systems. In IEEE FOCS 2013.

[45] Y. T. Lee and A. Sidford. 2014. Path Finding Methods for Linear Programming: Solving Linear Programs in $\tilde{O}$ (vrank) Iterations and Faster Algorithms for Maximum Flow. In FOCS.

[46] Bingdong Li, Jeff Springer, George Bebis, and Mehmet Hadi Gunes. 2013. A survey of network flow applications. Journal of Network and Computer Applications 36, 2 (2013), 567-581.

[47] Aleksander Madry. 2010. Fast approximation algorithms for cut-based problems in undirected graphs. In IEEE FOCS 2010. 245-254.

[48] A. Madry. 2013. Navigating Central Path with Electrical Flows: From Flows to Matchings, and Back. In FOCS

[49] Aleksander Madry. 2016. Computing Maximum Flow with Augmenting Electrical Flows. In IEEE FOCS 2016. 593-602.

[50] Y. Nesterov and A. Nemirovskii. 1994. Interior-Point Polynomial Algorithms in Convex Programming. Society for Industrial and Applied Mathematics.

[51] L. Orecchia, S. Sachdeva, and N. K. Vishnoi. 2012. Approximating the exponential, the lanczos method and an $\tilde{O}(m)$-time spectral algorithm for balanced separator.. In STOC.

[52] James B. Orlin. 2013. Max flows in $O(n m)$ time, or better. In Symposium on Theory of Computing Conference, STOC'13. 765-774.

[53] Bo Peng, Lei Zhang, and David Zhang. 2013. A survey of graph theoretical approaches to image segmentation. Pattern Recognition 46, 3 (2013), 1020-1038.

[54] Richard Peng. 2016. Approximate undirected maximum flows in $O(m$ polylog $(n))$ time. In ACM-SIAM SODA 2016. 1862-1867.

[55] Richard Peng and Daniel A. Spielman. 2014. An Efficient Parallel Solver for SDD Linear Systems. In ACM STOC 2014. 333-342.

[56] Harald Racke. 2008. Optimal hierarchical decompositions for congestion minimization in networks. In ACM STOC 2008. 255-264.

[57] L. I. Rudin, S. Osher, and E. Fatemi. 1992. Nonlinear total variation based noise removal algorithms. Physica D: nonlinear phenomena 60, 1-4 (1992), 259-268.

[58] Thatchaphol Saranurak and Di Wang. 2019. Expander Decomposition and Pruning: Faster, Stronger, and Simpler. In ACM-SIAM SODA 2019. 2616-2635.

[59] Aaron Schild. 2018. An almost-linear time algorithm for uniform random spanning tree generation. In ACM STOC 2018. 214-227.

[60] Alexander Schrijver. 2002. On the history of the transportation and maximum flow problems. Math. Program. 91, 3 (2002), 437-445

[61] J. Sherman. 2013. Nearly Maximum Flows in Nearly Linear Time. In FOCS 2013.

[62] J. Sherman. 2017. Area-convexity, $l_{\infty}$ regularization, and undirected multicommodity flow. In ACM STOC 2017.

[63] J. Sherman. 2017. Generalized Preconditioning and Undirected Minimum-cost Flow. In ACM-SIAM SODA 2017.

[64] Daniel D Sleator and Robert Endre Tarjan. 1983. A data structure for dynamic trees. Fournal of computer and system sciences 26, 3 (1983), 362-391.

[65] Daniel Dominic Sleator and Robert Endre Tarjan. 1985. Self-Adjusting Binary Search Trees. 7. ACM 32, 3 (1985), 652-686.

[66] D.A. Spielman and S. Teng. 2004. Nearly-linear Time Algorithms for Graph Partitioning, Graph Sparsification, and Solving Linear Systems. In STOC.

[67] D. Spielman and S. Teng. 2014. Nearly Linear Time Algorithms for Preconditioning and Solving Symmetric, Diagonally Dominant Linear Systems. SIAM $\mathcal{7}$. Matrix Anal. Appl. 35, 3 (2014), 835-885.

[68] Mingqiang Zhu, Stephen J Wright, and Tony F Chan. 2010. Duality-based algorithms for total-variation-regularized image restoration. Computational Optimization and Applications 47, 3 (2010), 377-400. 